\title{
SECOND-ORDER NECESSARY AND SUFFICIENT OPTIMALITY CONDITIONS FOR OPTIMIZATION PROBLEMS AND APPLICATIONS TO CONTROL THEORY*
}

\author{
EDUARDO CASAS ${ }^{\dagger}$ AND FREDI TRÖLTZSCH ${ }^{\ddagger}$
}

\begin{abstract}
This paper deals with a class of nonlinear optimization problems in a function space, where the solution is restricted by pointwise upper and lower bounds and by finitely many equality and inequality constraints of functional type. Second-order necessary and sufficient optimality conditions are established, where the cone of critical directions is arbitrarily close to the form which is expected from the optimization in finite dimensional spaces. The results are applied to some optimal control problems for ordinary and partial differential equations.
\end{abstract}

Key words. necessary and sufficient optimality conditions, control of differential equations, state constraints

AMS subject classifications. 49K20, 35J25, 90C45, 90C48

PII. S1052623400367698

1. Introduction. Let $(X, \mathcal{S}, \mu)$ be a measure space with $\mu(X)<+\infty$. In this paper we will study the following optimization problem:

$$
\begin{cases}\operatorname{minimize} J(u), & \\ u_{a}(x) \leq u(x) \leq u_{b}(x) & \text { a.e. } x \in X, \\ G_{j}(u)=0, & 1 \leq j \leq m_{1}, \\ G_{j}(u) \leq 0, & m_{1}+1 \leq j \leq m,\end{cases}
$$

where $u_{a}, u_{b} \in L^{\infty}(X)$ and $J, G_{j}: L^{\infty}(X) \longrightarrow \mathbb{R}$ are given functions with differentiability properties to be fixed later. We will state necessary and sufficient optimality conditions for a local minimum of $(\mathrm{P})$. Our main goal is to reduce the classical gap between the necessary and sufficient conditions for optimization problems in Banach spaces. We shall prove some optimality conditions very close to the ones for finite dimensional optimization problems. In the case of finite dimensions, strongly active inequality constraints (i.e., with strictly positive Lagrange multipliers) are considered in the critical cone by associated linearized equality constraints. Roughly speaking, this is what we are able to extend to infinite dimensions. Due to the lack of compactness, the classical proof of the sufficiency theorem known for finite dimensions cannot be transferred to the case of general Banach spaces. Our direct method of proof is able to overcome this difficulty. To our best knowledge, this result has not yet been presented in the literature. Of course, the bound constraints $u_{a}(x) \leq u(x) \leq u_{b}(x)$ introduce some additional difficulties in the study because they constitute an infinite number of constraints. In section 2 we introduce a slightly stronger regularity assumption than that considered in the Kuhn-Tucker theorem, which allows us to deal with the bound constraints.

\footnotetext{
*Received by the editors February 8, 2000; accepted for publication (in revised form) February 7, 2002; published electronically September 24, 2002.

http://www.siam.org/journals/siopt/13-2/36769.html

${ }^{\dagger}$ Departamento de Matemática Aplicada y Ciencias de la Computación, E.T.S.I. Industriales y de Telecomunicación, Universidad de Cantabria, 39005 Santander, Spain (eduardo.casas@unican.es). This author was partially supported by Dirección General de Enseñanza Superior e Investigación Científica (Spain).

${ }^{\ddagger}$ Fakultät für Mathematik, Technische Universität Chemnitz, D-09107 Chemnitz, Germany (f.troeltzsch@mathematik.tu-chemnitz.de).
} 
In section 4 we discuss the application of our general results to different types of optimal control problems. We consider the control of ODEs as well as that of partial differential equations of elliptic and parabolic type.

2. Necessary optimality conditions. In this section we will assume that $\bar{u}$ is a local solution of $(\mathrm{P})$, which means that there exists a real number $r>0$ such that for every feasible point of $(\mathrm{P})$, with $\|u-\bar{u}\|_{L^{\infty}(X)}<r$, we have that $J(\bar{u}) \leq J(u)$.

For every $\varepsilon>0$, we denote the set of points at which the bound constraints are $\varepsilon$-inactive by

$$
X_{\varepsilon}=\left\{x \in X: u_{a}(x)+\varepsilon \leq \bar{u}(x) \leq u_{b}(x)-\varepsilon\right\} .
$$

We make the following regularity assumption:

$$
\left\{\begin{array}{l}
\exists \varepsilon_{\bar{u}}>0 \text { and }\left\{h_{j}\right\}_{j \in I_{0}} \subset L^{\infty}(X), \quad \text { with supp } h_{j} \subset X_{\varepsilon_{\bar{u}}}, \\
\text { such that } G_{i}^{\prime}(\bar{u}) h_{j}=\delta_{i j}, \quad i, j \in I_{0}
\end{array}\right.
$$

where

$$
I_{0}=\left\{j \leq m \mid G_{j}(\bar{u})=0\right\} .
$$

$I_{0}$ is the set of indices corresponding to active constraints. We also denote the set of nonactive constraints by $I_{-}$

$$
I_{-}=\left\{j \leq m \mid G_{j}(\bar{u})<0\right\} .
$$

Obviously (2.1) is equivalent to the independence of the derivatives $\left\{G_{j}^{\prime}(\bar{u})\right\}_{j \in I_{0}}$ in $L^{\infty}\left(X_{\varepsilon_{\bar{u}}}\right)$. Under this assumption we can derive the first-order necessary conditions for optimality satisfied by $\bar{u}$. For the proof, the reader is referred to Bonnans and Casas [3] or Clarke [10].

THEOREM 2.1. Let us assume that (2.1) holds and that $J$ and $\left\{G_{j}\right\}_{j=1}^{m}$ are of class $C^{1}$ in a neighborhood of $\bar{u}$. Then there exist real numbers $\left\{\bar{\lambda}_{j}\right\}_{j=1}^{m} \subset \mathbb{R}$ such that

$$
\begin{aligned}
\bar{\lambda}_{j} \geq 0, \quad m_{1}+1 \leq j \leq m, \quad \bar{\lambda}_{j}=0 \quad \text { if } j \in I_{-}, \\
\left\langle J^{\prime}(\bar{u})+\sum_{j=1}^{m} \bar{\lambda}_{j} G_{j}^{\prime}(\bar{u}), u-\bar{u}\right\rangle \geq 0 \quad \forall u_{a} \leq u \leq u_{b} .
\end{aligned}
$$

Since we want to establish some optimality conditions useful for the study of control problems, we need to take into account the two-norm discrepancy; for this question, see, for instance, Ioffe [17] and Maurer [19]. Then we have to impose some additional assumptions on the functions $J$ and $G_{j}$.

(A1) There exist functions $f, g_{j} \in L^{2}(X), 1 \leq j \leq m$, such that for every $h \in$ $L^{\infty}(X)$

$$
J^{\prime}(\bar{u}) h=\int_{X} f(x) h(x) d \mu(x) \text { and } G_{j}^{\prime}(\bar{u}) h=\int_{X} g_{j}(x) h(x) d \mu(x), \quad 1 \leq j \leq m .
$$

(A2) If $\left\{h_{k}\right\}_{k=1}^{\infty} \subset L^{\infty}(X)$ is bounded, $h \in L^{\infty}(X)$, and $h_{k}(x) \rightarrow h(x)$ a.e. in $X$, then

$$
\left[J^{\prime \prime}(\bar{u})+\sum_{j=1}^{m} \bar{\lambda}_{j} G_{j}^{\prime \prime}(\bar{u})\right] h_{k}^{2} \rightarrow\left[J^{\prime \prime}(\bar{u})+\sum_{j=1}^{m} \bar{\lambda}_{j} G_{j}^{\prime \prime}(\bar{u})\right] h^{2} .
$$


If we define

$$
L(u, \lambda)=J(u)+\sum_{j=1}^{m} \lambda_{j} G_{j}(u) \quad \text { and } \quad d(x)=f(x)+\sum_{j=1}^{m} \bar{\lambda}_{j} g_{j}(x),
$$

then

$$
\frac{\partial L}{\partial u}(\bar{u}, \bar{\lambda}) h=\left[J^{\prime}(\bar{u})+\sum_{j=1}^{m} \bar{\lambda}_{j} G_{j}^{\prime}(\bar{u})\right] h=\int_{X} d(x) h(x) d \mu(x) \quad \forall h \in L^{\infty}(X) .
$$

From (2.3) we deduce that

$$
d(x)=\left\{\begin{array}{cl}
0 & \text { for almost every } x \in X, \text { where } u_{a}(x)<\bar{u}(x)<u_{b}(x) \\
\geq 0 & \text { for almost every } x \in X, \text { where } \bar{u}(x)=u_{a}(x) \\
\leq 0 & \text { for almost every } x \in X, \text { where } \bar{u}(x)=u_{b}(x)
\end{array}\right.
$$

Associated with $d$, we set

$$
X^{0}=\{x \in X:|d(x)|>0\}
$$

Given $\left\{\bar{\lambda}_{j}\right\}_{j=1}^{m}$ by Theorem 2.1, we define the cone of critical directions $(2.10)$

$C_{\bar{u}}^{0}=\left\{h \in L^{\infty}(X)\right.$ satisfying (2.11) and $h(x)=0$ for almost every $\left.x \in X^{0}\right\}$,

with

$$
\left\{\begin{array}{l}
G_{j}^{\prime}(\bar{u}) h=0 \text { if }\left(j \leq m_{1}\right) \text { or }\left(j>m_{1}, G_{j}(\bar{u})=0, \text { and } \bar{\lambda}_{j}>0\right), \\
G_{j}^{\prime}(\bar{u}) h \leq 0 \text { if } j>m_{1}, G_{j}(\bar{u})=0, \text { and } \bar{\lambda}_{j}=0, \\
h(x)= \begin{cases}\geq 0 & \text { if } \bar{u}(x)=u_{a}(x), \\
\leq 0 & \text { if } \bar{u}(x)=u_{b}(x) .\end{cases}
\end{array}\right.
$$
tions.

In the following theorem we state the necessary second-order optimality condi-

Theorem 2.2. Assume that (2.1), (A1), and (A2) hold; $\left\{\bar{\lambda}_{j}\right\}_{j=1}^{m}$ are the Lagrange multipliers satisfying (2.2) and (2.3); and $J$ and $\left\{G_{j}\right\}_{j=1}^{m}$ are of class $C^{2}$ in a neighborhood of $\bar{u}$. Then the following inequality is satisfied:

$$
\frac{\partial^{2} L}{\partial u^{2}}(\bar{u}, \bar{\lambda}) h^{2} \geq 0 \quad \forall h \in C_{\bar{u}}^{0} .
$$

To prove this theorem we will make use of the following lemma.

LEMMA 2.3. Let us assume that (2.1) holds and that $J$ and $\left\{G_{j}\right\}_{j=1}^{m}$ are of class $C^{2}$ in a neighborhood of $\bar{u}$. Let $h \in L^{\infty}(X)$ satisfy $G_{j}^{\prime}(\bar{u}) h=0$ for every $j \in I$, where $I$ is an arbitrary subset of $I_{0}$. Then there exist a number $\varepsilon_{h}>0$ and $C^{2}$-functions $\gamma_{j}:\left(-\varepsilon_{h},+\varepsilon_{h}\right) \longrightarrow \mathbb{R}, j \in I$, such that

$$
\left\{\begin{array}{l}
G_{j}\left(u_{t}\right)=0, j \in I, \quad \text { and } G_{j}\left(u_{t}\right)<0, j \notin I_{0}, \quad \forall|t| \leq \varepsilon_{h} \\
\gamma_{j}(0)=\gamma_{j}^{\prime}(0)=0, \quad j \in I
\end{array}\right.
$$

with $u_{t}=\bar{u}+t h+\sum_{j \in I} \gamma_{j}(t) h_{j},\left\{h_{j}\right\}_{j \in I}$ given by (2.1). 
Proof. Let $k$ be the cardinal number of $I$ and let us define $\omega: \mathbb{R} \times \mathbb{R}^{k} \longrightarrow \mathbb{R}^{k}$ by

$$
\omega(t, \rho)=\left(G_{j}\left(\bar{u}+t h+\sum_{i \in I} \rho_{i} h_{i}\right)\right)_{j \in I} .
$$

Then $\omega$ is of class $C^{2}$ in a neighborhood of $(0,0)$,

$$
\frac{\partial \omega}{\partial t}(0,0)=\left(G_{j}^{\prime}(\bar{u}) h\right)_{j \in I}=0 \quad \text { and } \quad \frac{\partial \omega}{\partial \rho}(0,0)=\left(G_{j}^{\prime}(\bar{u}) h_{i}\right)_{i, j \in I}=\text { identity } .
$$

Therefore we can apply the implicit function theorem and deduce the existence of $\varepsilon>0$ and functions $\gamma_{j}:(-\varepsilon,+\varepsilon) \longrightarrow \mathbb{R}$ of class $C^{2}, j \in I$, such that

$$
\omega(t, \gamma(t))=\omega(0,0)=0 \quad \forall t \in(-\varepsilon,+\varepsilon) \text { and } \gamma(0)=0,
$$

where $\gamma(t)=\left(\gamma_{j}(t)\right)_{j \in I}$. Furthermore, by differentiation in the previous identity we get

$$
\frac{\partial \omega}{\partial t}(0,0)+\frac{\partial \omega}{\partial \rho}(0,0) \gamma^{\prime}(0)=0 \Longrightarrow \gamma^{\prime}(0)=0 .
$$

Taking into account the continuity of $\gamma$ and $G_{j}$ and that $\gamma(0)=0$, we deduce the existence of $\varepsilon_{h} \leq \varepsilon$ such that (2.13) holds for every $t \in\left(-\varepsilon_{h},+\varepsilon_{h}\right)$.

Proof of Theorem 2.2. Let us take $h \in C_{\bar{u}}^{0}$ satisfying

$$
h(x)=0 \quad \text { if } u_{a}(x)<\bar{u}(x)<u_{a}(x)+\varepsilon \text { or } u_{b}(x)-\varepsilon<\bar{u}(x)<u_{b}(x)
$$

for some $\varepsilon \in\left(0, \varepsilon_{\bar{u}}\right]$. We introduce

$$
I=\left\{1, \ldots, m_{1}\right\} \cup\left\{j: m_{1}+1 \leq j \leq m, G_{j}(\bar{u})=0 \text {, and } G_{j}^{\prime}(\bar{u}) h=0\right\} .
$$

$I$ includes all equality constraints, all strongly active inequality constraints (i.e., $\bar{\lambda}_{j}>$ 0 ), and, depending on $h$, possibly some of the weakly active inequality constraints (i.e., $\bar{\lambda}_{j}=0$ ). Then we are under the assumptions of Lemma 2.3. Let us set

$$
u_{t}=\bar{u}+t h+\sum_{j \in I} \gamma_{j}(t) h_{j}, \quad t \in\left(-\varepsilon_{h}, \varepsilon_{h}\right)
$$

From Lemma 2.3 we know that $G_{j}\left(u_{t}\right)=0$ if $j \in I$, and $G_{j}\left(u_{t}\right)<0$ if $j \notin I_{0}$, provided that $t \in\left(-\varepsilon_{h},+\varepsilon_{h}\right)$. From (2.11) we deduce that $G_{j}(\bar{u})=0$ and $G_{j}^{\prime}(\bar{u}) h<0$ for $j \in I_{0} \backslash I$. Therefore we have that $G_{j}\left(u_{t}\right)<0$ for every $j \notin I$ and $t \in\left(0, \varepsilon_{0}\right)$, for some $\varepsilon_{0}>0$ small. On the other hand, the assumptions on $h$, along with the additional condition (2.14) and the fact that $\operatorname{supp} h_{j} \subset X_{\varepsilon_{\bar{u}}}$, imply that $u_{a}(x) \leq u_{t}(x) \leq u_{b}(x)$ for $t \geq 0$ small enough. Consequently, by taking $\varepsilon_{0}>0$ sufficiently small, we get that $u_{t}$ is a feasible control for $(\mathrm{P})$ for every $t \in\left[0, \varepsilon_{0}\right)$. Now we know $G_{j}\left(u_{t}\right)=0$ for $j \in I$ and $\bar{\lambda}_{j}=0$ for $j \notin I_{0}$ (cf. (2.2)). According to (2.11) we require $G_{j}^{\prime}(\bar{u}) h=0$ for active inequalities with $\bar{\lambda}_{j}>0$; hence if $i$ belongs to $I_{0} \backslash I$, then $\bar{\lambda}_{j}=0$ must hold. This leads to

$$
\sum_{j=1}^{m} \bar{\lambda}_{j} G_{j}\left(u_{t}\right)=0 \quad \forall t \in\left[0, \varepsilon_{0}\right) .
$$


Therefore the function $\phi:\left[0,+\epsilon_{0}\right) \longrightarrow \mathbb{R}$ given by

$$
\phi(t)=J\left(u_{t}\right)+\sum_{j=1}^{m} \bar{\lambda}_{j} G_{j}\left(u_{t}\right)
$$

has a local minimum at 0 and, taking into account that $\gamma_{j}^{\prime}(0)=0$,

$$
\begin{aligned}
\phi^{\prime}(0) & =\left(J^{\prime}(\bar{u})+\sum_{j=1}^{m} \bar{\lambda}_{j} G_{j}^{\prime}(\bar{u})\right)\left(h+\sum_{j \in I} \gamma_{j}^{\prime}(0) h_{j}\right) \\
& =\left[J^{\prime}(\bar{u})+\sum_{j=1}^{m} \bar{\lambda}_{j} G_{j}^{\prime}(\bar{u})\right] h=\int_{X} d(x) h(x) d \mu(x)=0 .
\end{aligned}
$$

The last identity follows from the fact that $h$ vanishes on $X^{0}$. Since the first derivative of $\phi$ is zero, the following second-order necessary optimality condition must hold:

$$
\begin{aligned}
0 \leq \phi^{\prime \prime}(0) & =\left[J^{\prime \prime}(\bar{u})+\sum_{j=1}^{m} \bar{\lambda}_{j} G_{j}^{\prime \prime}(\bar{u})\right] h^{2}+\left[J^{\prime}(\bar{u})+\sum_{j=1}^{m} \bar{\lambda}_{j} G_{j}^{\prime}(\bar{u})\right]\left(\sum_{i \in I} \gamma_{i}^{\prime \prime}(0) h_{i}\right) \\
& =\left[J^{\prime \prime}(\bar{u})+\sum_{j=1}^{m} \bar{\lambda}_{j} G_{j}^{\prime \prime}(\bar{u})\right] h^{2}+\sum_{i \in I} \gamma_{i}^{\prime \prime}(0) \int_{X} d(x) h_{i}(x) d \mu(x) \\
& =\left[J^{\prime \prime}(\bar{u})+\sum_{j=1}^{m} \bar{\lambda}_{j} G_{j}^{\prime \prime}(\bar{u})\right] h^{2}=\frac{\partial^{2} L}{\partial u^{2}}(\bar{u}, \bar{\lambda}) h^{2} .
\end{aligned}
$$

Here we have used (A1). Now let us consider $h \in L^{\infty}(X)$ satisfying (2.11) but not (2.14), i.e., $h$ is any critical direction. The main idea in this case is to approach $h$ by functions $h_{\varepsilon}$, which belong to the critical cone $C_{\bar{u}}^{0}$ and satisfy (2.14) as well. Then for every $\varepsilon>0$, we define $A_{\varepsilon}=X_{\varepsilon} \cup\left\{x \in X: \bar{u}(x)=u_{a}(x)\right.$ or $\left.\bar{u}(x)=u_{b}(x)\right\}$. This is the complement of the set of points $x$ satisfying (2.14). Set

$$
h_{\varepsilon}=h \chi_{A_{\varepsilon}}+\sum_{i \in I}\left[\int_{X \backslash A_{\varepsilon}} g_{i}(x) h(x) d \mu(x)\right] h_{i}=h \chi_{A_{\varepsilon}}+\hat{h},
$$

where $\chi_{A_{\varepsilon}}$ is the characteristic function of $A_{\varepsilon}$ and $I$ is given by (2.15). We verify that $h_{\varepsilon}$ belongs to $C_{\bar{u}}^{0}$, while $h \chi_{A_{\varepsilon}}$ is possibly not contained in this cone.

Thus for every $j \in I$, using (2.1) and taking $0<\varepsilon<\varepsilon_{\bar{u}}$, we have

$$
\begin{aligned}
G_{j}^{\prime}(\bar{u}) h_{\varepsilon}= & \int_{X} g_{j}(x)\left(h \chi_{A_{\varepsilon}}\right)(x) d \mu(x)+\int_{X} g_{j}(x) \hat{h}(x) d \mu(x) \\
= & \int_{A_{\varepsilon}} g_{j}(x) h(x) d \mu(x) \\
& +\sum_{i \in I}\left[\int_{X \backslash A_{\varepsilon}} g_{i}(x) h(x) d \mu(x)\right] \int_{X} g_{j}(x) h_{i}(x) d \mu(x) \\
= & \int_{A_{\varepsilon}} g_{j}(x) h(x) d \mu(x)+\sum_{i \in I}\left[\int_{X \backslash A_{\varepsilon}} g_{i}(x) h(x) d \mu(x)\right] \delta_{j i} \\
= & \int_{X} g_{j}(x) h(x) d \mu(x)=G_{j}^{\prime}(\bar{u}) h=0 .
\end{aligned}
$$


In the case of $j \in I_{0} \backslash I$, we have $G_{j}^{\prime}(\bar{u}) h<0$. Then it is enough to take $\varepsilon$ sufficiently small to get $G_{j}^{\prime}(\bar{u}) h_{\varepsilon}<0$.

Thus, recalling that supp $h_{j} \subset X_{\varepsilon_{\bar{u}}}$, we infer that $h_{\varepsilon}$ satisfies the conditions (2.11) and (2.14); therefore (2.12) holds for each $h_{\varepsilon}, \varepsilon>0$ small enough.

Finally, it is clear that $h_{\varepsilon}(x) \rightarrow h(x)$ a.e. in $X$ as $\varepsilon \rightarrow 0$. Therefore, assumption (A2) allows us to pass to the limit in the second-order optimality conditions satisfied for every $h_{\varepsilon}$ and to conclude (2.12).

3. Sufficient optimality conditions. Whenever nonlinear optimal control problems are solved, second-order sufficient conditions play an essential role in the numerical analysis. For instance, they ensure local convergence of Lagrange-Newton-SQP methods; see Alt and Malanowski [2], Dontchev et al. [11], Ito and Kunisch [18], or Schulz [23], and the references cited therein. Such conditions are important for error estimates as well. We refer, for instance, to Arada, Casas, and Tröltzsch [1] and Hager [15]. Finally, we mention that second-order conditions should be checked numerically to verify local optimality of computed solutions; see Mittelmann [21].

In this section, $\bar{u}$ is a given feasible element for the problem (P). Motivated again by the considerations on the two-norm discrepancy, we have to make some assumptions involving the $L^{\infty}(X)$ and $L^{2}(X)$ norms, as follows.

(A3) There exists a positive number $r>0$ such that $J$ and $\left\{G_{j}\right\}_{j=1}^{m}$ are of class $C^{2}$ in the $L^{\infty}(X)$-ball $B_{r}(\bar{u})$, and for every $\eta>0$ there exists $\varepsilon \in(0, r)$ such that for each $u \in B_{r}(\bar{u}),\|v-\bar{u}\|_{L^{\infty}(X)}<\varepsilon, h, h_{1}, h_{2} \in L^{\infty}(X)$, and $1 \leq j \leq m$ we have

$$
\left\{\begin{array}{l}
\left|\left[\frac{\partial^{2} L}{\partial u^{2}}(v, \bar{\lambda})-\frac{\partial^{2} L}{\partial u^{2}}(\bar{u}, \bar{\lambda})\right] h^{2}\right| \leq \eta\|h\|_{L^{2}(X)}^{2}, \\
\left|J^{\prime}(u) h\right| \leq M_{0,1}\|h\|_{L^{2}(X)}, \quad\left|G_{j}^{\prime}(u) h\right| \leq M_{j, 1}\|h\|_{L^{2}(X)} \\
\left|J^{\prime \prime}(u) h_{1} h_{2}\right| \leq M_{0,2}\left\|h_{1}\right\|_{L^{2}(X)}\left\|h_{2}\right\|_{L^{2}(X)}, \\
\left|G_{j}^{\prime \prime}(u) h_{1} h_{2}\right| \leq M_{j, 2}\left\|h_{1}\right\|_{L^{2}(X)}\left\|h_{2}\right\|_{L^{2}(X)} .
\end{array}\right.
$$

Analogously to (2.9) and (2.10), we define for every $\tau>0$

$$
\begin{aligned}
& X^{\tau}=\{x \in X:|d(x)|>\tau\}, \\
& C_{\bar{u}}^{\tau}=\left\{h \in L^{\infty}(X) \text { satisfying (2.11) and } h(x)=0 \text { a.e. } x \in X^{\tau}\right\} .
\end{aligned}
$$

The next theorem provides the second-order sufficient optimality conditions of (P). Although they seem to be different from the classical ones, we will prove later that they are equivalent; see Theorem 3.2 and Corollary 3.3.

THEOREM 3.1. Let $\bar{u}$ be a feasible point for problem $(\mathrm{P})$ verifying the first-order necessary conditions (2.2) and (2.3), and let us suppose that assumptions (2.1), (A1), and (A3) hold. Let us also assume that for every $h \in L^{\infty}(X)$ satisfying (2.11)

$$
\frac{\partial^{2} L}{\partial u^{2}}(\bar{u}, \bar{\lambda}) h^{2} \geq \delta_{1}\|h\|_{L^{2}\left(X \backslash X^{\tau}\right)}^{2}-\delta_{2}\|h\|_{L^{2}\left(X^{\tau}\right)}^{2}
$$

holds for some $\delta_{1}>0, \delta_{2} \geq 0$, and $\tau>0$. Then there exist $\varepsilon>0$ and $\delta>0$ such that $J(\bar{u})+\delta\|u-\bar{u}\|_{L^{2}(X)}^{2} \leq J(u)$ for every feasible point $u$ for $(\mathrm{P})$, with $\|u-\bar{u}\|_{L^{\infty}(X)}<\varepsilon$.

Proof. (i) Condition (3.4) is stable w.r.t. perturbations of $\bar{u}$. Without loss of generality, we will assume that $\delta_{2}>0$. From (A3) we deduce the existence of $r_{0} \in$ 
$(0, r)$ such that for all $h \in L^{\infty}(X)$ and $\|v-\bar{u}\|_{L^{\infty}(X)}<r_{0}$

$$
\left|\left[\frac{\partial^{2} L}{\partial u^{2}}(v, \bar{\lambda})-\frac{\partial^{2} L}{\partial u^{2}}(\bar{u}, \bar{\lambda})\right] h^{2}\right| \leq \min \left\{\frac{\delta_{1}}{2}, \delta_{2}\right\}\|h\|_{L^{2}(X)}^{2} .
$$

From this inequality and (3.4) it follows easily that

$$
\frac{\partial^{2} L}{\partial u^{2}}(v, \bar{\lambda}) h^{2} \geq \frac{\delta_{1}}{2}\|h\|_{L^{2}\left(X \backslash X^{\tau}\right)}^{2}-2 \delta_{2}\|h\|_{L^{2}\left(X^{\tau}\right)}^{2}
$$

for every $h$ satisfying (2.11) and $\|v-\bar{u}\|_{L^{\infty}(X)}<r_{0}$.

(ii) Some technical definitions. Let us set

$$
\begin{aligned}
& M=M_{0,2}+\sum_{j=1}^{m}\left|\bar{\lambda}_{j}\right| M_{j, 2} \quad \text { and } \quad \rho=\min \left\{1, \frac{\delta_{1}}{16 M}\right\} \\
& C_{1}=\max \left\{\frac{\delta_{1}}{4}, 2 \delta_{2}\right\}+\frac{3 M}{2}+\frac{4 M^{2}}{\delta_{1}}, \quad C_{2}=\frac{C_{1}}{2} \max _{j \in I_{0}}\left\|h_{j}\right\|_{L^{2}(X)}^{2}\left[\sum_{j=1}^{m} M_{j, 2}\right]^{2} \\
& C_{3}=2 C_{1} m \mu(X)^{1 / 2} \max _{j \in I_{0}}\left\|h_{j}\right\|_{L^{2}(X)}^{2} \max _{1 \leq j \leq m} M_{j, 1}
\end{aligned}
$$

Finally, we take

$$
\varepsilon=\min \left\{r_{0}, \sqrt{\frac{\delta_{1}}{64 C_{2} \mu(X)}}, \frac{8 \tau}{\delta_{1}+16 \delta_{2}}, \frac{\rho}{C_{3}} \min _{j \in I_{+}, j>m_{1}} \bar{\lambda}_{j}\right\},
$$

where

$$
I_{+}=\left\{1, \ldots, m_{1}\right\} \cup\left\{j>m_{1}: G_{j}(\bar{u})=0 \text { and } \bar{\lambda}_{j}>0\right\} .
$$

(iii) Approximation of $u-\bar{u}$ by elements of the critical cone. Let $u$ be a feasible point for problem $(\mathrm{P})$, with $\|u-\bar{u}\|_{L^{\infty}(X)}<\epsilon$. Then $u-\bar{u}$ will not, in general, belong to the critical cone. Therefore, we use the representation $u-\bar{u}=h+h_{0}$, where $h$ is in the critical cone and $h_{0}$ is some small correction.

Let us introduce the set of indices

$$
I_{u}=\left\{j \in I_{0}: G_{j}^{\prime}(\bar{u})(u-\bar{u})>0 \text { or }\left[G_{j}^{\prime}(\bar{u})(u-\bar{u})<0 \text { and } j \in I_{+}\right]\right\} .
$$

This is the set of indices for which we need to correct $G_{j}^{\prime}(\bar{u})(u-\bar{u})$, since the conditions of the critical cone are not met. We need to carry out this correction for equality constraints if $G_{j}^{\prime}(\bar{u})(u-\bar{u}) \neq 0$. We also need to apply this correction for an active inequality constraint satisfying $G_{j}^{\prime}(\bar{u})(u-\bar{u})>0$ or for a strongly active inequality constraint if $G_{j}^{\prime}(\bar{u})(u-\bar{u})<0$ holds. We define for all $j \in I_{u}$

$$
\alpha_{j}=G_{j}^{\prime}(\bar{u})(u-\bar{u}), \quad h_{0}=\sum_{j \in I_{u}} \alpha_{j} h_{j}, \quad \text { and } \quad h=u-\bar{u}-h_{0},
$$

where the elements $h_{j}$ are introduced in assumption (2.1). Then $h$ satisfies (2.11). This is seen as follows:

$$
G_{j}^{\prime}(\bar{u}) h_{0}=\sum_{i \in I_{u}} \alpha_{i} G_{j}^{\prime}(\bar{u}) h_{i}=\sum_{i \in I_{u}} \alpha_{i} \delta_{j i} .
$$


If $j \notin I_{u}$, then $\delta_{j i}=0$ for all $i \in I_{u}$; hence

$$
G_{j}^{\prime}(\bar{u}) h=G_{j}^{\prime}(\bar{u})(u-\bar{u})-G_{j}^{\prime}(\bar{u}) h_{0}=G_{j}^{\prime}(\bar{u})(u-\bar{u}) \begin{cases}=0 & \text { if } j \leq m_{1}, \\ \leq 0 & \text { if } j>m_{1}\end{cases}
$$

(the last inequality follows from $\left.j \notin I_{u}\right)$. Thus $G_{j}^{\prime}(\bar{u}) h$ fulfills the conditions of the critical cone. If $j \in I_{u}$, then

$$
G_{j}^{\prime}(\bar{u}) h=G_{j}^{\prime}(\bar{u})(u-\bar{u})-\alpha_{j} \delta_{j j}=\alpha_{j}-\alpha_{j}=0,
$$

and $G_{j}^{\prime}(\bar{u}) h$ also fulfills the conditions of the critical cone.

Let us now estimate $h_{0}$ in $L^{2}(X)$. For every $j \in I_{u}$ there exists $v_{j}=\bar{u}+\theta_{j}(u-\bar{u})$, with $0<\theta_{j}<1$, such that

$$
0 \geq G_{j}(u)=G_{j}(\bar{u})+G_{j}^{\prime}(\bar{u})(u-\bar{u})+\frac{1}{2} G_{j}^{\prime \prime}\left(v_{j}\right)(u-\bar{u})^{2}=\alpha_{j}+\frac{1}{2} G_{j}^{\prime \prime}\left(v_{j}\right)(u-\bar{u})^{2} .
$$

If $\alpha_{j} \geq 0$, we deduce from (3.11) and (3.1) that

$$
\left|\alpha_{j}\right|=\alpha_{j} \leq \frac{1}{2}\left|G_{j}^{\prime \prime}\left(v_{j}\right)(u-\bar{u})^{2}\right| \leq \frac{1}{2} M_{j, 2}\|u-\bar{u}\|_{L^{2}(X)}^{2} .
$$

If $\alpha_{j}<0$ and $G_{j}(u)=0$, we get

$$
\left|\alpha_{j}\right|=-\alpha_{j}=\frac{1}{2} G_{j}^{\prime \prime}\left(v_{j}\right)(u-\bar{u})^{2} \leq \frac{1}{2} M_{j, 2}\|u-\bar{u}\|_{L^{2}(X)}^{2} .
$$

Let us define

$$
I_{u}^{-}=\left\{j \in I_{u}: G_{j}(u)<0 \text { and } \alpha_{j}<0\right\} .
$$

This is the set of all indices, where we do not obtain an estimate of $\alpha_{j}$ having the order $\|u-\bar{u}\|_{L^{2}(x)}^{2}$. We should notice at this point that $\bar{\lambda}_{j}>0$ holds for all $j \in I_{u}^{-}$. (Since $u$ must be feasible, $j$ stands for an inequality constraint. Therefore, $0>\alpha_{j}=$ $G_{j}^{\prime}(\bar{u})(u-\bar{u})$, and $j \in I_{u}$ implies $j \in I_{+}$.) Then we have

$$
\left\|h_{0}\right\|_{L^{2}(X)} \leq \max _{j \in I_{0}}\left\|h_{j}\right\|_{L^{2}(X)}\left[\frac{1}{2}\left(\sum_{j=1}^{m} M_{j, 2}\right)\|u-\bar{u}\|_{L^{2}(X)}^{2}+\sum_{j \in I_{u}^{-}}\left|\alpha_{j}\right|\right] .
$$

(iv) Estimation of $J(u)-J(\bar{u})$. Using (2.6), (2.7), (3.6), (3.10), and (3.11), for some $v=\bar{u}+\theta(u-\bar{u}), 0<\theta<1$,

$$
\begin{aligned}
J(u) & =J(u)+\sum_{j=1}^{m_{1}} \bar{\lambda}_{j} G_{j}(u)+\sum_{j=m_{1}+1}^{m} \bar{\lambda}_{j} G_{j}(u)-\sum_{j=m_{1}+1}^{m} \bar{\lambda}_{j} G_{j}(u) \\
& =L(u, \bar{\lambda})-\sum_{j=m_{1}+1}^{m} \bar{\lambda}_{j} G_{j}(u) \\
& \geq L(u, \bar{\lambda})-\sum_{j \in I_{u}^{-}} \bar{\lambda}_{j} G_{j}(u) \geq L(u, \bar{\lambda})-\rho \sum_{j \in I_{u}^{-}} \bar{\lambda}_{j} G_{j}(u)
\end{aligned}
$$


holds, since $\rho<1$. Therefore,

$$
\begin{aligned}
J(u) \geq & L(u, \bar{\lambda})-\rho \sum_{j \in I_{u}^{-}} \bar{\lambda}_{j} G_{j}(u)=L(\bar{u}, \bar{\lambda})+\frac{\partial L}{\partial u}(\bar{u}, \bar{\lambda})(u-\bar{u})+\frac{1}{2} \frac{\partial^{2} L}{\partial u^{2}}(v, \bar{\lambda})(u-\bar{u})^{2} \\
& -\rho \sum_{j \in I_{u}^{-}} \bar{\lambda}_{j} \alpha_{j}-\frac{\rho}{2} \sum_{j \in I_{u}^{-}} \bar{\lambda}_{j} G_{j}^{\prime \prime}\left(v_{j}\right)(u-\bar{u})^{2} \\
= & J(\bar{u})+\int_{X} d(x)(u(x)-\bar{u}(x)) d \mu(x)+\frac{1}{2} \frac{\partial^{2} L}{\partial u^{2}}(v, \bar{\lambda}) h^{2} \\
& +\frac{\partial^{2} L}{\partial u^{2}}(v, \bar{\lambda}) h h_{0}+\frac{1}{2} \frac{\partial^{2} L}{\partial u^{2}}(v, \bar{\lambda}) h_{0}^{2}+\rho \sum_{j \in I_{u}^{-}} \bar{\lambda}_{j}\left|\alpha_{j}\right|-\frac{\rho}{2} \sum_{j \in I_{u}^{-}} \bar{\lambda}_{j} G_{j}^{\prime \prime}\left(v_{j}\right)(u-\bar{u})^{2} .
\end{aligned}
$$

Now from (2.8), (2.11), (3.1), (3.5), and (3.6) it follows that

$$
\begin{aligned}
J(u) \geq & J(\bar{u})+\tau \int_{X^{\tau}}|u(x)-\bar{u}(x)| d \mu(x)+\frac{\delta_{1}}{4}\|h\|_{L^{2}\left(X \backslash X^{\tau}\right)}^{2}-\delta_{2}\|h\|_{L^{2}\left(X^{\tau}\right)}^{2} \\
& -M\left\|h_{0}\right\|_{L^{2}(X)}\|h\|_{L^{2}(X)}-\frac{M}{2}\left\|h_{0}\right\|_{L^{2}(X)}^{2}+\rho \sum_{j \in I_{u}^{-}} \bar{\lambda}_{j}\left|\alpha_{j}\right| \\
& -\frac{\rho}{2}\left[\sum_{j \in I_{u}^{-}} \bar{\lambda}_{j} M_{j, 2}\right]\|u-\bar{u}\|_{L^{2}(X)}^{2} \\
\geq & J(\bar{u})+\frac{\tau}{\varepsilon}\|u-\bar{u}\|_{L^{2}\left(X^{\tau}\right)}^{2}+\frac{\delta_{1}}{8}\|u-\bar{u}\|_{L^{2}\left(X \backslash X^{\tau}\right)}^{2}-\frac{\delta_{1}}{4}\left\|h_{0}\right\|_{L^{2}\left(X \backslash X^{\tau}\right)}^{2} \\
& -2 \delta_{2}\|u-\bar{u}\|_{L^{2}\left(X^{\tau}\right)}^{2}-2 \delta_{2}\left\|h_{0}\right\|_{L^{2}\left(X^{\tau}\right)}^{2} \\
& -M\left\|h_{0}\right\|_{L^{2}(X)}\left(\|u-\bar{u}\|_{L^{2}(X)}+\left\|h_{0}\right\|_{L^{2}(X)}\right)-\frac{M}{2}\left\|h_{0}\right\|_{L^{2}(X)}^{2} \\
& +\rho \sum_{j \in I_{u}^{-}} \bar{\lambda}_{j}\left|\alpha_{j}\right|-\frac{\rho}{2} M\|u-\bar{u}\|_{L^{2}(X)}^{2} .
\end{aligned}
$$

Using the definition of $\varepsilon$ from (3.9), we have

$$
\frac{\tau}{\varepsilon}-2 \delta_{2} \geq \frac{\delta_{1}}{8}
$$

On the other hand,

$$
\begin{aligned}
M\left\|h_{0}\right\|_{L^{2}(X)}\|u-\bar{u}\|_{L^{2}(X)} & =2\left[\frac{\sqrt{\delta_{1}}}{4}\|u-\bar{u}\|_{L^{2}(X)}\right]\left[\frac{2 M}{\sqrt{\delta_{1}}}\left\|h_{0}\right\|_{L^{2}(X)}\right] \\
& \leq \frac{\delta_{1}}{16}\|u-\bar{u}\|_{L^{2}(X)}^{2}+\frac{4 M^{2}}{\delta_{1}}\left\|h_{0}\right\|_{L^{2}(X)}^{2} .
\end{aligned}
$$

From the definitions of $C_{1}$ and $\rho$ given in (3.7) and (3.6) along with (3.15), (3.16), and (3.17), we get

$$
\begin{aligned}
J(u) \geq & J(\bar{u})+\frac{\delta_{1}}{8}\|u-\bar{u}\|_{L^{2}(X)}^{2}-C_{1}\left\|h_{0}\right\|_{L^{2}(X)}^{2} \\
& -\frac{\delta_{1}}{16}\|u-\bar{u}\|_{L^{2}(X)}^{2}+\rho \sum_{j \in I_{u}^{-}} \bar{\lambda}_{j}\left|\alpha_{j}\right|-\frac{\delta_{1}}{32}\|u-\bar{u}\|_{L^{2}(X)}^{2} \\
= & J(\bar{u})+\frac{\delta_{1}}{32}\|u-\bar{u}\|_{L^{2}(X)}^{2}-C_{1}\left\|h_{0}\right\|_{L^{2}(X)}^{2}+\rho \min _{j \in I_{+}, j>m_{1}} \bar{\lambda}_{j} \sum_{j \in I_{u}^{-}}\left|\alpha_{j}\right| .
\end{aligned}
$$


(v) Two auxiliary estimates and final result. From (3.7), (3.9), and (3.14) we get, on using $(a+b)^{2} \leq 2\left(a^{2}+b^{2}\right)$,

$$
\begin{aligned}
C_{1}\left\|h_{0}\right\|_{L^{2}(X)}^{2} & \leq C_{1} \max _{j \in I_{0}}\left\|h_{j}\right\|_{L^{2}(X)}^{2}\left[\frac{1}{2}\left(\sum_{j=1}^{m} M_{j, 2}\right)^{2}\|u-\bar{u}\|_{L^{2}(X)}^{4}+2\left(\sum_{j \in I_{u}^{-}}\left|\alpha_{j}\right|\right)^{2}\right] \\
& =C_{2}\|u-\bar{u}\|_{L^{2}(X)}^{4}+2 C_{1} \max _{j \in I_{0}}\left\|h_{j}\right\|_{L^{2}(X)}^{2}\left(\sum_{j \in I_{u}^{-}}\left|\alpha_{j}\right|\right)^{2} \\
& \leq C_{2} \varepsilon^{2} \mu(X)\|u-\bar{u}\|_{L^{2}(X)}^{2}+2 C_{1} \max _{j \in I_{0}}\left\|h_{j}\right\|_{L^{2}(X)}^{2}\left(\sum_{j \in I_{u}^{-}}\left|\alpha_{j}\right|\right)^{2} \\
& \leq \frac{\delta_{1}}{64}\|u-\bar{u}\|_{L^{2}(X)}^{2}+2 C_{1} \max _{j \in I_{0}}\left\|h_{j}\right\|_{L^{2}(X)}^{2}\left(\sum_{j \in I_{u}^{-}}\left|\alpha_{j}\right|\right)^{2} .
\end{aligned}
$$

The definition of $\alpha_{j}$ given by (3.10) along with assumption (3.1) imply

$$
\left|\alpha_{j}\right| \leq M_{j, 1}\|u-\bar{u}\|_{L^{2}(X)} \leq M_{j, 1} \varepsilon \sqrt{\mu(X)} .
$$

From (3.8) and the above inequality, we deduce

$$
2 C_{1} \max _{j \in I_{0}}\left\|h_{j}\right\|_{L^{2}(X)}^{2}\left(\sum_{j \in I_{u}^{-}}\left|\alpha_{j}\right|\right) \leq C_{3} \varepsilon .
$$

Definition (3.9) and (3.21) lead to

$$
\min _{j \in I_{+}, j>m_{1}} \bar{\lambda}_{j}-2 C_{1} \max _{j \in I_{0}}\left\|h_{j}\right\|_{L^{2}(X)}^{2}\left(\sum_{j \in I_{u}^{-}}\left|\alpha_{j}\right|\right) \geq 0 .
$$

Finally, combining (3.18), (3.19), and (3.22), we conclude the desired result:

$$
J(u) \geq J(\bar{u})+\frac{\delta_{1}}{64}\|u-\bar{u}\|_{L^{2}(X)}^{2} .
$$

Now we prove the equivalence between the sufficient optimality conditions stated in Theorem 3.1 and the classical ones.

TheOrem 3.2. Let $\bar{u}$ be a feasible point of $(\mathrm{P})$ satisfying (2.2) and (2.3). Let $C_{\bar{u}}$ be the set of elements $h \in L^{\infty}(X)$ satisfying (2.11), and $C_{\bar{u}}^{\tau}$ be given by (3.3). Let us suppose that assumptions (2.1), (A1), and (A3) hold. Let $\tau>0$ be given. Then the following statements are equivalent:

$$
\begin{gathered}
\exists \delta>0: \frac{\partial^{2} L}{\partial u^{2}}(\bar{u}, \bar{\lambda}) h^{2} \geq \delta\|h\|_{L^{2}(X)}^{2} \quad \forall h \in C_{\bar{u}}^{\tau}, \\
\exists \delta_{1}>0, \delta_{2} \geq 0: \frac{\partial^{2} L}{\partial u^{2}}(\bar{u}, \bar{\lambda}) h^{2} \geq \delta_{1}\|h\|_{L^{2}\left(X \backslash X^{\tau}\right)}^{2}-\delta_{2}\|h\|_{L^{2}\left(X^{\tau}\right)}^{2} \quad \forall h \in C_{\bar{u}} .
\end{gathered}
$$

Proof. It is obvious that (3.24) implies (3.23), since $h=0$ in $X^{\tau}$ if $h \in C_{\bar{u}}^{\tau}$. Therefore, it is enough to take $\delta=\delta_{1}$. Let us prove the opposite implication. Let $h \in C_{\bar{u}}$. We set $h_{\tau}=h \chi_{X^{\tau}}$, where $\chi_{X^{\tau}}$ is the characteristic function of $X^{\tau}$ and

$$
I_{h}=\left\{j \in I_{0}: G_{j}^{\prime}(\bar{u})\left(h-h_{\tau}\right)>0 \text { or }\left[G_{j}^{\prime}(\bar{u})\left(h-h_{\tau}\right)<0 \text { and } G_{j}^{\prime}(\bar{u}) h=0\right]\right\} .
$$


We define

$$
\alpha_{j}=G_{j}^{\prime}(\bar{u})\left(h-h_{\tau}\right) \quad \forall j \in I_{h}, \quad \hat{h}=\sum_{j \in I_{h}} \alpha_{j} h_{j}, \quad \text { and } \quad h_{0}=h-h_{\tau}-\hat{h},
$$

where the functions $h_{j}$ are given by (2.1).

Let us see that $h_{0} \in C_{\bar{u}}^{\tau}$. Since $\operatorname{supp} h_{j} \subset X_{\varepsilon_{\bar{u}}}$ and $h-h_{\tau}=h\left(1-\chi_{X^{\tau}}\right)$, we have that $h_{0}(x)=0$ for $x \in X^{\tau}$. Now we distinguish between the cases $j \in I_{h}$ and $j \in I_{0} \backslash I_{h}$.

If $j \in I_{h}$, then

$$
G_{j}^{\prime}(\bar{u}) h_{0}=G_{j}^{\prime}(\bar{u})\left(h-h_{\tau}\right)-\sum_{i \in I_{h}} \alpha_{i} G_{j}^{\prime}(\bar{u}) h_{i}=G_{j}^{\prime}(\bar{u})\left(h-h_{\tau}\right)-\alpha_{j}=0 .
$$

If $j \in I_{0} \backslash I_{h}$, then from the definition of $I_{h}$ we obtain that $G_{j}^{\prime}(\bar{u}) h_{0}=G_{j}^{\prime}(\bar{u})(h-$ $\left.h_{\tau}\right) \leq 0$.

If this inequality reduces to an equality $G_{j}^{\prime}(\bar{u})\left(h-h_{\tau}\right)=0$, then $h_{0}$ verifies that the condition is in $C_{\bar{u}}^{\tau}$. In the remaining case in which $j \in I_{0} \backslash I_{h}$ but $G_{j}^{\prime}(\bar{u})\left(h-h_{\tau}\right)<0$, using again the definition of $I_{h}$, we deduce that $G_{j}^{\prime}(\bar{u}) h<0 . \quad\left(G_{j}^{\prime}(\bar{u}) h=0\right.$ and $G_{j}^{\prime}(\bar{u})\left(h-h_{\tau}\right)<0$ would give $j \in I_{h}$. $)$ Consequently, since $h \in C_{\bar{u}}$, we have that $j>m_{1}$ and $\bar{\lambda}_{j}=0$ (otherwise, $h \in C_{\bar{u}}^{\tau}$ and $\bar{\lambda}_{j}>0$ would imply $G_{j}^{\prime}(\bar{u}) h=0$ ). Then the inequality $G_{j}^{\prime}(\bar{u}) h_{0}<0$ also means that $h_{0}$ shows the condition to be in $C_{\bar{u}}^{\tau}$.

We now prove that

$$
\|\hat{h}\|_{L^{2}(X)} \leq C_{0}\left\|h_{\tau}\right\|_{L^{2}(X)},
$$

where

$$
C_{0}=\sum_{j \in I_{0}}\left\|g_{j}\right\|_{L^{2}(X)}\left\|h_{j}\right\|_{L^{2}(X)}
$$

$g_{j}$ being given in (2.4). Indeed, if $\alpha_{j}>0$, then

$\left|\alpha_{j}\right|=\alpha_{j}=G_{j}^{\prime}(\bar{u})\left(h-h_{\tau}\right)=G_{j}^{\prime}(\bar{u}) h-G_{j}^{\prime}(\bar{u}) h_{\tau} \leq-G_{j}^{\prime}(\bar{u}) h_{\tau} \leq\left\|g_{j}\right\|_{L^{2}(X)}\left\|h_{\tau}\right\|_{L^{2}(X)}$.

If $\alpha_{j}<0$, then from the definition of $I_{h}$ we have that $G_{j}^{\prime}(\bar{u}) h=0$; therefore

$$
\left|\alpha_{j}\right|=-\alpha_{j}=-G_{j}^{\prime}(\bar{u})\left(h-h_{\tau}\right)=G_{j}^{\prime}(\bar{u}) h_{\tau} \leq\left\|g_{j}\right\|_{L^{2}(X)}\left\|h_{\tau}\right\|_{L^{2}(X)} .
$$

Combining the previous two inequalities and the definition of $\hat{h}$, we get (3.25).

Finally, taking $M$ as in (3.6), we obtain from (3.23) and (3.25)

$$
\begin{aligned}
\frac{\partial^{2} L}{\partial u^{2}}(\bar{u}, \bar{\lambda}) h^{2}= & \frac{\partial^{2} L}{\partial u^{2}}(\bar{u}, \bar{\lambda}) h_{0}^{2}+\frac{\partial^{2} L}{\partial u^{2}}(\bar{u}, \bar{\lambda})\left(h_{\tau}+\hat{h}\right)^{2}+2 \frac{\partial^{2} L}{\partial u^{2}}(\bar{u}, \bar{\lambda}) h_{0}\left(h_{\tau}+\hat{h}\right) \\
\geq & \delta\left\|h_{0}\right\|_{L^{2}(X)}^{2}-M\left\|h_{\tau}+\hat{h}\right\|_{L^{2}(X)}^{2}-2 M\left\|h_{0}\right\|_{L^{2}(X)}\left\|h_{\tau}+\hat{h}\right\|_{L^{2}(X)} \\
\geq & \frac{\delta}{2}\left\|h-h_{\tau}\right\|_{L^{2}(X)}^{2}-\delta\|\hat{h}\|_{L^{2}(X)}^{2}-2 M\left(\left\|h_{\tau}\right\|_{L^{2}(X)}^{2}+\|\hat{h}\|_{L^{2}(X)}^{2}\right) \\
& -2 M\left(\left\|h-h_{\tau}\right\|_{L^{2}(X)}+\|\hat{h}\|_{L^{2}(X)}\right)\left(\left\|h_{\tau}\right\|_{L^{2}(X)}+\|\hat{h}\|_{L^{2}(X)}\right) \\
\geq & \frac{\delta}{2}\left\|h-h_{\tau}\right\|_{L^{2}(X)}^{2}-C_{0}^{2} \delta\left\|h_{\tau}\right\|_{L^{2}(X)}^{2}-2 M\left(C_{0}^{2}+1\right)\left\|h_{\tau}\right\|_{L^{2}(X)}^{2} \\
& -2 M\left(C_{0}+1\right)\left(\left\|h-h_{\tau}\right\|_{L^{2}(X)}+C_{0}\left\|h_{\tau}\right\|_{L^{2}(X)}\right)\left\|h_{\tau}\right\|_{L^{2}(X)}
\end{aligned}
$$




$$
\begin{aligned}
\geq & \frac{\delta}{4}\left\|h-h_{\tau}\right\|_{L^{2}(X)}^{2} \\
& -\left\{C_{0}^{2} \delta+2 M\left(C_{0}^{2}+1\right)+\frac{4 M^{2}\left(C_{0}+1\right)^{2}}{\delta}+2 M\left(C_{0}+1\right) C_{0}\right\}\left\|h_{\tau}\right\|_{L^{2}(X)}^{2} \\
= & \delta_{1}\|h\|_{L^{2}\left(X \backslash X^{\tau}\right)}^{2}-\delta_{2}\|h\|_{L^{2}\left(X^{\tau}\right)}^{2},
\end{aligned}
$$

where obviously $\delta_{1}>0$ and $\delta_{2} \geq 0$ are independent of $h \in C_{\bar{u}}$.

The following corollary is an immediate consequence of Theorems 3.1 and 3.2.

Corollary 3.3. Let $\bar{u}$ be a feasible point for problem (P) satisfying (2.2) and (2.3), and suppose that assumptions (2.1), (A1), and (A3) hold. Assume also that

$$
\frac{\partial^{2} L}{\partial u^{2}}(\bar{u}, \bar{\lambda}) h^{2} \geq \delta\|h\|_{L^{2}(X)}^{2} \quad \forall h \in C_{\bar{u}}^{\tau}
$$

for some $\delta>0$ and $\tau>0$ given. Then there exist $\varepsilon>0$ and $\alpha>0$ such that $J(\bar{u})+\alpha\|u-\bar{u}\|_{L^{2}(X)}^{2} \leq J(u)$ for every feasible point $u$ for $(\mathrm{P})$, with $\|u-\bar{u}\|_{L^{\infty}(X)}<\varepsilon$.

Remark 3.4. Comparing the sufficient optimality condition (3.4) with the necessary condition (2.12), we notice the existence of a gap between the two, arising from two facts. First, the constant $\delta_{1}$ is strictly positive in (3.4), and it can be zero in (2.12), which is the classical situation even in finite dimensions. Second, we cannot substitute, in general, $C_{\bar{u}}^{\tau}$, with $\tau>0$, for $C_{\bar{u}}^{0}$ in (3.26), as is done in (2.12), because of the presence of an infinite number of constraints. Quite similar strategies are employed by Maurer and Zowe [20], Maurer [19], Donchev et al. [11], and Dunn [12]. The following example, due to Dunn [13], demonstrates the impossibility of taking $\tau=0$ in (3.26). Let us consider $X=[0,1], \mathcal{S}$ the $\sigma$-algebra of Lebesgue-measurable sets of $[0,1], \mu$ the Lebesgue measure in $[0,1]$, and $a(x)=1-2 x$. The optimization problem is

$$
\left\{\begin{array}{l}
\operatorname{minimize} J(u)=\int_{0}^{1}\left[2 a(x) u(x)-\operatorname{sign}(a(x)) u(x)^{2}\right] d x \\
u \in L^{\infty}([0,1]), u(x) \geq 0 \text { a.e. } x \in[0,1] .
\end{array}\right.
$$

Let us set $\bar{u}(x)=\max \{0,-a(x)\}$. Then we have that

$$
J^{\prime}(\bar{u}) h=\int_{0}^{1} 2[a(x)-\operatorname{sign}(a(x)) \bar{u}(x)] h(x) d x=\int_{0}^{1 / 2} 2 a(x) h(x) d x \geq 0
$$

holds for all $h \in L^{2}([0,1])$, with $h(x) \geq 0$. If we assume that $h(x)=0$ for $x \in X^{0}$,

$J^{\prime \prime}(\bar{u}) h^{2}=-\int_{0}^{1} 2 \operatorname{sign}(a(x)) h^{2}(x) d x=2 \int_{1 / 2}^{1} h^{2}(x) d x-2 \int_{0}^{1 / 2} h^{2}(x) d x=2\|h\|_{L^{2}(X)}^{2}$

holds, where, following the notation introduced in (2.9),

$$
X^{0}=\{x \in[0,1]:|d(x)|>0\}=\left[0, \frac{1}{2}\right) .
$$

Thus (3.26) holds with $\delta=2$ and $\tau=0$. However, $\bar{u}$ is not a local minimum in $L^{\infty}([0,1])$. Indeed, let us take for $0<\varepsilon<\frac{1}{2}$

$$
u_{\varepsilon}(x)=\left\{\begin{array}{cl}
\bar{u}(x)+3 \varepsilon & \text { if } x \in\left[\frac{1}{2}-\varepsilon, \frac{1}{2}\right] \\
\bar{u}(x) & \text { otherwise }
\end{array}\right.
$$


Then we have $J\left(u_{\varepsilon}\right)-J(\bar{u})=-3 \varepsilon^{3}<0$. The reader can easily check that the only points $u$ satisfying the first-order optimality conditions are given by the formula

$$
u(x)=\left\{\begin{array}{cl}
0 & \text { if } x \in Z, \\
\operatorname{sign}(a(x)) a(x) & \text { otherwise }
\end{array}\right.
$$

where $Z$ is any measurable subset of $[0,1]$ satisfying that $a(x) \geq 0$ for every $x \in Z$. None of these points is a local minimum of the optimization problem. Moreover, if we define $u_{k}(x)=k \cdot \max \{0, a(x)\}$, then $J\left(u_{k}\right)=k(2-k) / 6 \rightarrow-\infty$ when $k \rightarrow+\infty$.

\section{Application to some optimal control problems.}

4.1. An abstract control problem. Let, in addition to the measure space $(X, S, \mu), Y$ and $Z$ be real Banach spaces; let $A: Y \rightarrow Z$ be a linear continuous operator; and let $B: Y \times L^{\infty}(X) \rightarrow Z$ be an operator of class $C^{2}$. Moreover, $F, F_{j}: Y \times L^{\infty}(X) \rightarrow \mathbb{R}$ are functionals of class $C^{2}, j=1, \ldots, m$. Consider the optimal control problem

$$
\begin{cases}\operatorname{minimize} F(y, u) & \\ A y+B(y, u)=0 & \\ u_{a}(x) \leq u(x) \leq u_{b}(x) & \text { a.e. } x \in X \\ F_{j}(y, u)=0, & 1 \leq j \leq m_{1} \\ F_{j}(y, u) \leq 0, & m_{1}+1 \leq j \leq m\end{cases}
$$

where the control $u$ is taken from $L^{\infty}(X)$. We assume that for all $u \in L^{\infty}(X)$ the equation $A y+B(y, u)=0$ admits a unique solution $y \in Y$, so that a control-state mapping $G: u \mapsto y$ is defined. Moreover, the inverse operator $\left(A+\frac{\partial B}{\partial y}(y, u)\right)^{-1}$ : $Z \rightarrow Y$ is assumed to exist for all $(y, u) \in Y \times L^{\infty}(X)$ as a linear continuous operator. Then the implicit function theorem yields that $G$ is of class $C^{2}$ from $L^{\infty}(X)$ to $Y$. The first- and second-order derivatives $G^{\prime}(u)$ and $G^{\prime \prime}(u)$ are given as follows: Define $y=G(u), z_{h}=G^{\prime}(u) h$, and $z_{h_{1} h_{2}}:=G^{\prime \prime}(u)\left[h_{1}, h_{2}\right]:=\left(G^{\prime \prime}(u) h_{1}\right) h_{2}$. Then $z_{h}$ is the unique solution of

$$
A z+\frac{\partial B}{\partial y}(y, u) z+\frac{\partial B}{\partial u}(y, u) h=0
$$

while $z_{h_{1} h_{2}}$ is uniquely determined by

$$
\left\{\begin{aligned}
A z+\frac{\partial B}{\partial y}(y, u) z=- & \left\{\frac{\partial^{2} B}{\partial y^{2}}(y, u)\left[z_{h_{1}}, z_{h_{2}}\right]+\frac{\partial^{2} B}{\partial y \partial u}(y, u)\left[z_{h_{1}}, h_{2}\right]\right. \\
& \left.+\frac{\partial^{2} B}{\partial u \partial y}(y, u)\left[h_{1}, z_{h_{2}}\right]+\frac{\partial^{2} B}{\partial u^{2}}(y, u)\left[h_{1}, h_{2}\right]\right\}
\end{aligned}\right.
$$

We omit the proof, which can easily be transferred from that of Theorem 2.3 in [7]. The abstract control problem (OC) fits in the optimization problem (P) by

$$
J(u):=F(G(u), u), \quad G_{j}(u):=F_{j}(G(u), u) .
$$

In this way, we obtain necessary and/or sufficient conditions for local solutions $(\bar{y}, \bar{u})$ of (OC) by application of Theorems 2.1, 2.2, and 3.1 and Corollary 3.3, provided that the corresponding assumptions (2.1) and (A1)-(A3) are satisfied. We tacitly assume 
this in what follows and formulate these results in a way that is convenient for optimal control problems. A Lagrange function $\mathcal{L}=\mathcal{L}(y, u, \varphi, \lambda)$ is associated with (OC) by

$$
\mathcal{L}(y, u, \varphi, \lambda)=F(y, u)-\langle\varphi, A y+B(y, u)\rangle+\sum_{j=1}^{m} \lambda_{j} F_{j}(y, u),
$$

where $\varphi \in Z^{*}$, and $\langle\cdot, \cdot\rangle$ denotes the duality between $Z$ and $Z^{*}$. Notice that we must distinguish between $L$ for $(\mathrm{P})$ and $\mathcal{L}$ for $(\mathrm{OC})$. We have

$$
J^{\prime}(\bar{u}) h=\frac{\partial F}{\partial y}(\bar{y}, \bar{u}) G^{\prime}(\bar{u}) h+\frac{\partial F}{\partial u}(\bar{y}, \bar{u}) h
$$

and obtain similar expressions for $G_{j}(\bar{u}) h$. Therefore, (2.6) yields

$$
\left\{\begin{aligned}
\frac{\partial L}{\partial u}(\bar{u}, \bar{\lambda}) h= & {\left[\frac{\partial F}{\partial y}(\bar{y}, \bar{u})+\sum_{j=1}^{m} \bar{\lambda}_{j} \frac{\partial F_{j}}{\partial y}(\bar{y}, \bar{u})\right] G^{\prime}(\bar{u}) h } \\
& +\left[\frac{\partial F}{\partial u}(\bar{y}, \bar{u})+\sum_{j=1}^{m} \bar{\lambda}_{j} \frac{\partial F_{j}}{\partial u}(\bar{y}, \bar{u})\right] h .
\end{aligned}\right.
$$

Define an adjoint state $\varphi \in Z^{*}$ by

$$
\left[\frac{\partial F}{\partial y}(\bar{y}, \bar{u})+\sum_{j=1}^{m} \bar{\lambda}_{j} \frac{\partial F_{j}}{\partial y}(\bar{y}, \bar{u})\right] y=\left\langle\bar{\varphi}, A y+\frac{\partial B}{\partial y}(\bar{y}, \bar{u}) y\right\rangle \quad \forall y \in Y .
$$

We assume that $\bar{\varphi}$ is well defined by (4.5), which is true in our applications. Notice that (4.5) is equivalent to $\partial \mathcal{L} / \partial y(\bar{y}, \bar{u}, \bar{\varphi}, \bar{\lambda}) y=0$ for all $y \in Y$; that is, $\partial \mathcal{L} / \partial y(\bar{y}, \bar{u}, \bar{\varphi}, \bar{\lambda})=$ 0 in the sense of $Y^{*}$. Insert $y=z_{h}=G^{\prime}(\bar{u}) h$ into (4.5); then $y$ solves (4.1), and the right-hand side of $(4.5)$ is equal to $-\langle\bar{\varphi}, \partial B / \partial u(\bar{y}, \bar{u}) h\rangle$. Substituting this for the first item in (4.4), we find that

$$
\frac{\partial L}{\partial u}(\bar{u}, \bar{\lambda}) h=\frac{\partial \mathcal{L}}{\partial u}(\bar{y}, \bar{u}, \bar{\varphi}, \bar{\lambda}) h
$$

for all $h \in L^{\infty}(X)$. If (A1) is satisfied, then we deduce from (2.7) that $d(x)$ expresses the derivative $\partial \mathcal{L} / \partial u$, i.e.,

$$
\frac{\partial \mathcal{L}}{\partial u}(\bar{y}, \bar{u}, \bar{\varphi}, \bar{\lambda}) h=\int_{X} d(x) h(x) d \mu(x) .
$$

Corollary 4.1. Define $J$ and $G_{j}, j=1, \ldots, m$, as above, and let $\bar{u}$ with associated state $\bar{y}$ be a local solution of (OC). If the regularity assumption (2.1) is fulfilled, then there are Lagrange multipliers $\bar{\lambda}_{j}, j=1, \ldots, m$, such that (2.2), (2.3) are satisfied. Assume further that $\bar{\varphi} \in Z^{*}$ is uniquely determined by (4.5). Then (2.3) is equivalent, with

$$
\frac{\partial \mathcal{L}}{\partial u}(\bar{y}, \bar{u}, \bar{\varphi}, \bar{\lambda})(u-\bar{u}) \geq 0 \quad \forall u_{a} \leq u \leq u_{b} .
$$

If additionally (A1) is satisfied, then $\frac{\partial \mathcal{L}}{\partial u}(\bar{y}, \bar{u}, \bar{\varphi}, \bar{\lambda})$ can be identified with a real function $d=d(x)$, and (4.8) admits the form

$$
\int_{X} d(x)(u(x)-\bar{u}(x)) \geq 0 \quad \forall u_{a} \leq u \leq u_{b}
$$


Proof. The statement follows from Theorem 2.1: The variational inequality (4.8) is obtained from (2.3) by (2.6) and (4.6). If (A1) is satisfied, then (4.8) and (4.7) imply (4.9).

Let us now apply the second-order conditions to the control system. We have to express $\partial^{2} L / \partial u^{2}$ in terms of $\mathcal{L}$. From

$$
L(u, \lambda)=F(G(u), u)+\sum_{j=1}^{m} \lambda_{j} F_{j}(G(u), u)
$$

we get, after some straightforward computations,

$$
\left\{\begin{aligned}
\frac{\partial^{2} L}{\partial u^{2}}(\bar{u}, \bar{\lambda})\left[h_{1}, h_{2}\right]= & {\left[F^{\prime \prime}(\bar{y}, \bar{u})+\sum_{j=1}^{m} \bar{\lambda}_{j} F_{j}^{\prime \prime}(\bar{y}, \bar{u})\right]\left[\left(y_{1}, h_{1}\right),\left(y_{2}, h_{2}\right)\right] } \\
& +\left[\frac{\partial F}{\partial y}(\bar{y}, \bar{u})+\sum_{j=1}^{m} \bar{\lambda}_{j} \frac{\partial F_{j}}{\partial y}(\bar{y}, \bar{u})\right] G^{\prime \prime}(\bar{u})\left[h_{1}, h_{2}\right],
\end{aligned}\right.
$$

where $y_{i}=G^{\prime}(\bar{u}) h_{i}=z_{h_{i}}, i=1,2$. We know that $G^{\prime \prime}(\bar{u})\left[h_{1}, h_{2}\right]=z_{h_{1} h_{2}}$, where $z=z_{h_{1} h_{2}}$ is the solution of (4.2); hence this term can be reduced to $z_{h_{1}}$ and $z_{h_{2}}$. By definition of $\bar{\varphi},(4.2)$, and (4.5),

$$
\left\{\begin{aligned}
{\left[\frac{\partial F}{\partial y}+\sum_{j=1}^{m} \bar{\lambda}_{j} \frac{\partial F_{j}}{\partial y}\right] z_{h_{1} h_{2}} } & =\left\langle\bar{\varphi}, A z_{h_{1} h_{2}}+\frac{\partial B}{\partial y} z_{h_{1} h_{2}}\right\rangle \\
& =-\left\langle\bar{\varphi}, B^{\prime \prime}(\bar{y}, \bar{u})\left[\left(z_{h_{1}}, h_{1}\right),\left(z_{h_{2}}, h_{2}\right)\right]\right\rangle
\end{aligned}\right.
$$

is obtained. Insert this into (4.10); then $y_{i}=z_{h_{i}}$ and $z_{h_{1} h_{2}}=G^{\prime \prime}(\bar{u})\left[h_{1}, h_{2}\right]$ give

$$
\left\{\begin{aligned}
\frac{\partial^{2} L}{\partial u^{2}}(\bar{u}, \bar{\lambda})\left[h_{1}, h_{2}\right]= & {\left[F^{\prime \prime}(\bar{y}, \bar{u})+\sum_{j=1}^{m} \bar{\lambda}_{j} F_{j}^{\prime \prime}(\bar{y}, \bar{u})\right]\left[\left(y_{1}, h_{1}\right),\left(y_{2}, h_{2}\right)\right] } \\
& -\left\langle\bar{\varphi}, B^{\prime \prime}(\bar{y}, \bar{u})\left[\left(y_{1}, h_{1}\right),\left(y_{2}, h_{2}\right)\right]\right\rangle \\
= & \mathcal{L}_{(y, u)}^{\prime \prime}(\bar{y}, \bar{u}, \bar{\varphi}, \bar{\lambda})\left[\left(y_{1}, h_{1}\right),\left(y_{2}, h_{2}\right)\right] .
\end{aligned}\right.
$$

Notice that in (4.11) the increments $\left(y_{i}, h_{i}\right)$ cannot be chosen independently, since $y_{i}$ and $h_{i}$ are coupled through $y_{i}=G^{\prime}(\bar{u}) h_{i}=z_{h_{i}}$. Hence the definition of $z_{h_{i}}$ shows that the pairs $(y, h)=\left(y_{i}, h_{i}\right)$ have to solve the linearized equation

$$
A y+\frac{\partial B}{\partial y}(\bar{y}, \bar{u}) y+\frac{\partial B}{\partial u}(\bar{y}, \bar{u}) h=0 .
$$

Corollary 4.2. Assume that (2.1), (A1), and (A2) are satisfied and that $\bar{\varphi} \in Z^{*}$ is uniquely defined by (4.5). Then

$$
\mathcal{L}_{(y, u)}^{\prime \prime}(\bar{y}, \bar{u}, \bar{\varphi}, \bar{\lambda})(y, h)^{2} \geq 0
$$

holds for all $(y, h) \in Y \times L^{\infty}(X)$ that satisfy the linearized equation (4.12) and the relations

$$
\begin{cases}\frac{\partial F_{j}}{\partial y}(\bar{y}, \bar{u}) y+\frac{\partial F_{j}}{\partial u}(\bar{y}, \bar{u}) h=0 & \text { if }\left(j \leq m_{1}\right) \\ & \text { or }\left(j>m_{1}, F_{j}(\bar{y}, \bar{u})=0, \text { and } \bar{\lambda}_{j}>0\right), \\ \frac{\partial F_{j}}{\partial y}(\bar{y}, \bar{u}) y+\frac{\partial F_{j}}{\partial u}(\bar{y}, \bar{u}) h \leq 0 & \text { if } j>m_{1}, F_{j}(\bar{y}, \bar{u})=0, \text { and } \bar{\lambda}_{j}=0\end{cases}
$$




$$
\begin{gathered}
h(x)=\left\{\begin{array}{lll}
\geq 0 & \text { if } & \bar{u}(x)=u_{a}(x), \\
\leq 0 & \text { if } & \bar{u}(x)=u_{b}(x),
\end{array}\right. \\
h(x)=0 \text { if } x \in X^{0} .
\end{gathered}
$$

The second-order sufficient optimality conditions are given by the following.

Corollary 4.3. Let $(\bar{y}, \bar{u})$ fulfill all constraints of (OC) and, together with $\bar{\varphi}$ and $\bar{\lambda}_{j}, j=1, \ldots, m$, the first-order optimality conditions stated in Corollary 4.1. Assume that (2.1), (A1), and (A3) hold true. If there exist $\tau>0, \delta_{1}>0$, and $\delta_{2}>0$ such that

$$
\mathcal{L}_{(y, u)}^{\prime \prime}(\bar{y}, \bar{u}, \bar{\varphi}, \bar{\lambda})(y, h)^{2} \geq \delta_{1}\|h\|_{L^{2}\left(X \backslash X^{\tau}\right)}^{2}-\delta_{2}\|h\|_{L^{2}\left(X^{\tau}\right)}^{2}
$$

holds for all $(y, h) \in Y \times L^{\infty}(X)$ that satisfy the linearized equation (4.12) and the relations (4.14), (4.15), then the conclusions of Theorem 3.1 hold true; hence $\bar{u}$ is a local solution of (OC). Here, the set $X^{\tau}$ is defined by (3.2). The same conclusion is true if the condition

$$
\mathcal{L}_{(y, u)}^{\prime \prime}(\bar{y}, \bar{u}, \bar{\varphi}, \bar{\lambda})(y, h)^{2} \geq \delta\|h\|_{L^{2}(X)}^{2}
$$

holds instead of (4.17) with some $\delta>0$, where $h(x)=0$ for all $x \in X^{\tau}$ for some $\tau>0$, and $(y, h)$ are subject to (4.12), (4.14), and (4.15).

4.2. Optimal control of ODEs. In this section we discuss an optimal control problem governed by an ODE. We concentrate on a very simplified setting to give the reader an easy insight into the application of the theory. For further problems, we refer to the book by Hestenes [16]. Define

$$
\begin{aligned}
F(y, u) & =\psi(y(T))+\int_{0}^{T} f_{0}(t, y(t), u(t)) d t, \\
F_{j}(y, u) & =\int_{0}^{T} f_{j}(t, y(t), u(t)) d t,
\end{aligned}
$$

$j=1, \ldots, m$, and consider the optimal control problem

$(\mathrm{ODE})$

$$
\begin{cases}\operatorname{minimize} F(y, u), & \\ y^{\prime}(t)+b(t, y(t), u(t))=0 & \text { a.e. } t \in(0, T), \\ y(0)=0, & \text { a.e. } t \in(0, T), \\ u_{a}(t) \leq u(t) \leq u_{b}(t) & 1 \leq j \leq m_{1}, \\ F_{j}(y, u)=0, & m_{1}+1 \leq j \leq m . \\ F_{j}(y, u) \leq 0, & \end{cases}
$$

Here, $T$ is a fixed time. To reduce the number of technicalities, let us discuss only real-valued functions $y$ and $u$. The vector-valued case can be handled analogously. For the same reason, we assume that the functions $\psi, f_{j}$, and $b$ are of class $C^{2}$ on $\mathbb{R}$ and $[0, T] \times \mathbb{R} \times\left[\min u_{a}, \max u_{b}\right]$, respectively, although weaker Carathéodory-type conditions would suffice. We introduce the state space $Y=\left\{y \in W^{1, \infty}(0, T) \mid y(0)=\right.$ $0\}$ and set

$$
(A y)(t)=y^{\prime}(t), \quad(B(y, u))(t)=b(t, y(t), u(t)) .
$$

$A$ is continuous from $Y$ to $Z=L^{\infty}(0, T)$, and $B$ is of class $C^{2}$ from $Y \times L^{\infty}(0, T)$ to Z. In this way, (ODE) is related to (OC) as a particular case, where $X=[0, T]$, and $\mu$ 
is the Lebesgue measure, $d \mu=d t$. For convenience, the variable $t \in X$ is substituted for the variable $x$, which was used in the former sections.

Let $(\bar{y}, \bar{u}) \in Y \times L^{\infty}(0, T)$ be our reference solution, a given candidate for optimality. For (ODE), the Lagrange function

$$
\mathcal{L}(y, u, \varphi, \lambda)=F(y, u)-\int_{0}^{T} \varphi\left(y^{\prime}+b(t, y, u)\right) d t+\sum_{j=1}^{m} \lambda_{j} F_{j}(y, u)
$$

is introduced, where $\varphi \in W^{1, \infty}(0, T)$ will be defined by the adjoint equation below. In an obvious way this $\varphi$ generates a linear functional belonging to $Z^{*}$, but it has more regularity than arbitrary functionals of this space.

Remark 4.4. Given the inhomogeneous initial condition $y(0)=y_{0}$, we have to work with the space $Y=W^{1, \infty}(0, T)$ and must include the initial condition in the definition of $A$. Then the additional term $\varphi_{0}\left(y(0)-y_{0}\right)$ would appear in (4.19). This requires some more notational effort. However, the optimality conditions are not changed. Therefore, without loss of generality we confine ourselves to a homogeneous initial condition.

Having in mind the particular form of $\varphi$, we see that here (4.5) is nothing more than the definition of the adjoint equation

$$
\left\{\begin{aligned}
-\varphi^{\prime}+\frac{\partial b}{\partial y}(t, \bar{y}, \bar{u}) \varphi & =\frac{\partial f_{0}}{\partial y}(t, \bar{y}, \bar{u})+\sum_{j=1}^{m} \bar{\lambda}_{j} \frac{\partial f_{j}}{\partial y}(t, \bar{y}, \bar{u}), \\
\varphi(T) & =\psi^{\prime}(y(T)) .
\end{aligned}\right.
$$

It is obvious that (4.20) admits a unique solution $\bar{\varphi} \in W^{1, \infty}(0, T)$. In section 5 we show that (A1) is satisfied for (ODE). We obtain the following derivatives of the Lagrange function:

$$
\frac{\partial \mathcal{L}}{\partial u}(\bar{y}, \bar{u}, \bar{\varphi}, \bar{\lambda}) h=\int_{0}^{T}\left(\frac{\partial f_{0}}{\partial u}-\bar{\varphi} \frac{\partial b}{\partial u}+\sum_{j=1}^{m} \bar{\lambda}_{j} \frac{\partial f_{j}}{\partial u} h\right) d t
$$

(all derivatives taken at $(\bar{y}, \bar{u})$ ); hence $\partial \mathcal{L} / \partial u$ can be identified with $d \in L^{\infty}(0, T)$,

$$
d(t)=\left(\frac{\partial f_{0}}{\partial u}-\bar{\varphi} \frac{\partial b}{\partial u}+\sum_{j=1}^{m} \bar{\lambda}_{j} \frac{\partial f_{j}}{\partial u}\right)(t) .
$$

The second derivative of $\mathcal{L}$ is

$$
\left\{\begin{array}{l}
\mathcal{L}_{(y, u)}^{\prime \prime}(\bar{y}, \bar{u}, \bar{\varphi}, \bar{\lambda})\left[\left(y_{1}, h_{1}\right),\left(y_{2}, h_{2}\right)\right]=\psi^{\prime \prime}(\bar{y}(T)) y_{1}(T) y_{2}(T) \\
\quad+\int_{0}^{T}\left\{\left(y_{1}, h_{1}\right)\left(f_{0}^{\prime \prime}(\bar{y}, \bar{u})-\bar{\varphi} b^{\prime \prime}(\bar{y}, \bar{u})+\sum_{j=1}^{m} \bar{\lambda}_{j} f_{j}^{\prime \prime}(\bar{y}, \bar{u})\right)\left(y_{2}, h_{2}\right)^{\top}\right\} d t
\end{array}\right.
$$

where $f_{0}^{\prime \prime}, b^{\prime \prime}, f_{j}^{\prime \prime}$ stand for $2 \times 2$ Hessian matrices taken at $(t, \bar{y}(t), \bar{u}(t))$. It is easy to verify that (A2) is satisfied.

The first-order necessary optimality conditions are stated in Corollary 4.1. In particular, the following variational inequality has to be satisfied:

$$
\int_{X} d(t)(u(t)-\bar{u}(t)) d t \geq 0
$$


for all $u_{a} \leq u(t) \leq u_{b}$; hence $\bar{u}(t)=u_{a}$, where $d(t)>0$, and $\bar{u}(t)=u_{b}$, where $d(t)<0$. (These points form the set $X^{0}$.) No information is obtained where $d$ is zero. Roughly speaking, this is the set for which higher-order conditions are needed.

The second-order necessary conditions are formulated in Corollary 4.2. We have to specify the linearized equation (4.12) and the form of the derivatives in the relations (4.14). The linearized equation is

$$
\left\{\begin{aligned}
y^{\prime}+\frac{\partial b}{\partial y}(t, \bar{y}, \bar{u}) y+\frac{\partial b}{\partial u}(t, \bar{y}, \bar{u}) h & =0, \\
y(0) & =0,
\end{aligned}\right.
$$

while

$$
\frac{\partial F_{j}}{\partial y}(\bar{y}, \bar{u}) y+\frac{\partial F_{j}}{\partial u}(\bar{y}, \bar{u}) h=\int_{X}\left\{\frac{\partial f_{j}}{\partial y}(t, \bar{y}, \bar{u}) y+\frac{\partial f_{j}}{\partial u}(t, \bar{y}, \bar{u}) h\right\} d t .
$$

4.3. Optimal boundary control of an elliptic equation. As a further application, we consider an elliptic control problem. For convenience, we discuss a simplified version and refer for further reading to [9].

Let $\Omega \subset \mathbb{R}^{N}$ be a bounded domain with boundary $\Gamma$ of class $C^{0,1}$. Let $\nu$ denote the outward unit normal vector at $\Gamma$, and $\partial_{\nu}$ be the associated normal derivative. Define

$$
\begin{aligned}
& F(y, u)=\int_{\Omega} \gamma_{0}(x, y(x)) d x+\int_{\Omega} \psi_{0}(x, y(x)) d \mu_{0}(x)+\int_{\Gamma} f_{0}(x, y(x), u(x)) d S(x), \\
& F_{j}(y, u)=\int_{\Omega} \gamma_{j}(x, y(x)) d x+\int_{\Omega} \psi_{j}(x, y(x)) d \mu_{j}(x)+\int_{\Gamma} f_{j}(x, y(x), u(x)) d S(x),
\end{aligned}
$$

$j=1, \ldots, m$. We assume that the functions $\gamma_{j}=\gamma_{j}(x, y), \psi_{j}=\psi_{j}(x, y)$, and $f_{j}=f_{j}(x, y, u)$ are of class $C^{2}$ on $\bar{\Omega} \times \mathbb{R}$ and $\bar{\Omega} \times \mathbb{R}^{2}$, respectively. Moreover, real Borel measures $\mu_{j}$ are given on $\Omega$. Here, $\mu$ is the Lebesgue surface measure induced on $\Gamma, d \mu=d S$. The appearance of the measures $\mu_{j}$ in the functionals will heavily influence the verification of assumptions (A1)-(A3). Therefore, the easier case $\psi_{j}=0$, $j=1, \ldots, m$, is of interest as well.

Consider the optimal control problem

$$
\begin{cases}\operatorname{minimize} F(y, u), & \\ -\Delta y+y=0 & \text { in } \Omega \\ \partial_{\nu} y+b(x, y, u)=0 & \text { on } \Gamma \\ u_{a}(x) \leq u(x) \leq u_{b}(x) & \text { a.e. on } \Gamma \\ F_{j}(y, u)=0, & 1 \leq j \leq m_{1} \\ F_{j}(y, u) \leq 0, & m_{1}+1 \leq j \leq m\end{cases}
$$

In this setting, the boundary control $u$ is looked upon in the space $L^{\infty}(\Gamma)$, hence $X=\Gamma$, while the state $y$ belongs to $Y=\left\{y \in H^{1}(\Omega) \mid-\Delta y+y \in L^{q}(\Omega), \partial_{\nu} y \in\right.$ $\left.L^{p}(\Gamma)\right\}$. (Here $q>N / 2$ and $p>N-1$ are given fixed.) Endowing $Y$ with the graph norm, it is known that $Y \subset C(\bar{\Omega})$, the embedding being continuous. Assume that $b=b(x, y, u)$ satisfies the same conditions as the $f_{j}$. Additionally, we require that $(\partial b / \partial y)(x, y, u) \geq 0$ on $\Gamma \times \mathbb{R} \times\left[\min u_{a}, \max u_{b}\right]$. Define

$$
A: Y \rightarrow L^{q}(\Omega) \times L^{p}(\Gamma) \quad \text { and } \quad B: Y \times L^{\infty}(\Gamma) \rightarrow L^{q}(\Omega) \times L^{p}(\Gamma)
$$


by

$$
(A y)=\left(\begin{array}{c}
-\Delta y+y \\
\partial_{\nu} y
\end{array}\right) \quad \text { and } \quad B(y, u)(x)=\left(\begin{array}{c}
0 \\
b(x, y(x), u(x))
\end{array}\right)
$$

The equation $A y+B(y, u)=0$, which is equivalent to our elliptic boundary value problem, admits for each $u \in L^{\infty}(\Gamma)$ exactly one solution $y \in Y$. The mapping $u \mapsto y$ is of class $C^{2}$ from $L^{\infty}(\Gamma)$ to $Y$. Now we proceed in the same way as in the preceding section. The Lagrange function is

$$
\begin{aligned}
\mathcal{L}(y, u, \varphi, \lambda)= & F(y, u)-\int_{\Omega}(-\Delta y+y) \varphi d x \\
& -\int_{\Gamma}\left(\partial_{\nu} y+b(x, y, u)\right) \varphi d S+\sum_{j=1}^{m} \lambda_{j} F_{j}(y, u),
\end{aligned}
$$

where $\varphi \in W^{1, s}(\Omega)$ for all $s<\frac{N}{N-1}$ is the adjoint state. The adjoint state $\varphi$ together with its trace $\varphi_{\mid \Gamma}$ forms a Lagrange multiplier of $Z^{*}=L^{q^{\prime}}(\Omega) \times L^{p^{\prime}}(\Gamma)$ having higher regularity. Here (4.5) reduces to the adjoint equation

$$
\left\{\begin{array}{c}
-\Delta \varphi+\varphi=\frac{\partial \gamma_{0}}{\partial y}+\left.\frac{\partial \psi_{0}}{\partial y} \mu_{0}\right|_{\Omega}+\sum_{j=1}^{m} \bar{\lambda}_{j}\left(\frac{\partial \gamma_{j}}{\partial y}+\left.\frac{\partial \psi_{j}}{\partial y} \mu_{j}\right|_{\Omega}\right) \\
\partial_{\nu} \varphi+\frac{\partial b}{\partial y} \varphi=\frac{\partial f_{0}}{\partial y}+\sum_{j=1}^{m} \bar{\lambda}_{j} \frac{\partial f_{j}}{\partial y}+\left.\frac{\partial \psi_{0}}{\partial y} \mu_{0}\right|_{\Gamma}+\left.\sum_{j=1}^{m} \bar{\lambda}_{j} \frac{\partial \psi_{j}}{\partial y} \mu_{j}\right|_{\Gamma}
\end{array}\right.
$$

(all partial derivatives taken at $(x, \bar{y}(x), \bar{u}(x))$ ). This equation has a unique solution $\bar{\varphi} \in W^{1, s}(\Omega)$ associated with $(\bar{y}, \bar{u}, \bar{\lambda})$. Notice that for $N=2$ the Sobolev imbedding theorem yields $\varphi \in L^{\sigma}(\Omega)$ for all $\sigma<\infty$, but not in general $\varphi \in L^{\infty}(\Omega)$. For $N \geq 3$ the regularity of $\varphi$ is even lower. This indicates that we have to discuss assumptions (A1)-(A3) with more care. We shall do this in the last section.

The situation is easier in the case $\psi_{j}=0, j=0, \ldots, m$. Then all data given in the adjoint equation are bounded and measurable, and the regularity theory of elliptic equations yields $\bar{\varphi} \in C(\bar{\Omega})$ (see $[5]$ ).

Let us establish the first- and second-order derivatives of $\mathcal{L}$. We get

$$
\frac{\partial \mathcal{L}}{\partial u}(\bar{y}, \bar{u}, \bar{\varphi}, \bar{\lambda}) h=\int_{\Gamma}\left(\frac{\partial f_{0}}{\partial u}(x, \bar{y}, \bar{u})+\sum_{j=1}^{m} \bar{\lambda}_{j} \frac{\partial f_{j}}{\partial u}(x, \bar{y}, \bar{u})-\bar{\varphi} \frac{\partial b}{\partial u}(x, \bar{y}, \bar{u})\right) h d S
$$

and

$$
\begin{aligned}
& \mathcal{L}_{(y, u)}^{\prime \prime}(\bar{y}, \bar{u}, \bar{\varphi}, \bar{\lambda})\left[\left(y_{1}, h_{1}\right),\left(y_{2}, h_{2}\right)\right] \\
& =\int_{\Gamma}\left(y_{1}, h_{1}\right)\left(f_{0}^{\prime \prime}(x, \bar{y}, \bar{u})+\sum_{j=1}^{m} \bar{\lambda}_{j} f_{j}^{\prime \prime}(x, \bar{y}, \bar{u})-\bar{\varphi} b^{\prime \prime}(x, \bar{y}, \bar{u})\right)\left(y_{2}, h_{2}\right)^{\top} d S \\
& \quad+\int_{\Omega}\left(\frac{\partial^{2} \gamma_{0}}{\partial y^{2}}(x, \bar{y})+\sum_{j=1}^{m} \bar{\lambda}_{j} \frac{\partial^{2} \gamma_{j}}{\partial y^{2}}(x, \bar{y})\right) y_{1} y_{2} d x \\
& \quad+\int_{\Omega} \frac{\partial^{2} \psi_{0}}{\partial y^{2}}(x, \bar{y}) y_{1} y_{2} d \mu_{0}+\sum_{j=1}^{m} \bar{\lambda}_{j} \frac{\partial^{2} \psi_{j}}{\partial y^{2}}(x, \bar{y}) y_{1} y_{2} d \mu_{j}
\end{aligned}
$$


We observe that, due to our notation, there is almost no difference in the expressions derived for the case of (ODE) in (4.21), (4.23). The first- and second-order conditions for our elliptic problem (ELL) admit the following form: Set

$$
d(x)=\frac{\partial f_{0}}{\partial u}(x, \bar{y}(x), \bar{u}(x))+\sum_{j=1}^{m} \bar{\lambda}_{j} \frac{\partial f_{j}}{\partial u}(x, \bar{y}(x), \bar{u}(x))-\bar{\varphi} \frac{\partial b}{\partial u}(x, \bar{y}(x), \bar{u}(x)) .
$$

Then $d$ has the same form as in (4.22). The first- and second-order optimality conditions are given by Corollaries 4.1-4.3. There we set $X=\Gamma$ to obtain all first- and second-order conditions for (ELL). Now the directions $(y, h)$ are coupled through the linearized boundary value problem

$$
\left\{\begin{array}{l}
-\Delta y+y=0 \\
\partial_{\nu} y+\frac{\partial b}{\partial y}(x, \bar{y}, \bar{u}) y+\frac{\partial b}{\partial u}(x, \bar{y}, \bar{u}) h=0
\end{array}\right.
$$

The derivatives in (4.14), (4.15) admit the form

$$
\left\{\begin{aligned}
\frac{\partial F_{j}}{\partial y}(\bar{y}, \bar{u}) y+\frac{\partial F_{j}}{\partial u}(\bar{y}, \bar{u}) h= & \int_{\Omega} \frac{\partial \gamma_{j}}{\partial y}(t, \bar{y}) y d x+\int_{\Omega} \frac{\partial \psi_{j}}{\partial y}(t, \bar{y}) y d \mu_{j} \\
& +\int_{\Gamma}\left\{\frac{\partial f_{j}}{\partial y}(t, \bar{y}, \bar{u}) y+\frac{\partial f_{j}}{\partial u}(t, \bar{y}, \bar{u}) h\right\} d S
\end{aligned}\right.
$$

In this way, we have obtained the second-order sufficient condition for a simplified elliptic control problem. For the discussion of more general problems, we refer to [7], [9]. We should underline again that so far we have stated the optimality condition in a formal way. It remains to verify (A1)-(A3) to make our theory work. Low regularity of the adjoint state $\varphi$ can be an essential obstacle for this. We refer to section 5 .

4.4. Optimal distributed control of a parabolic equation. We confine ourselves to a distributed parabolic control problem. A more general class, including boundary control and boundary observation, is considered in a separate paper by Raymond and Tröltzsch [22]. Let $\Omega$ be defined as in the last section, and set $Q=$ $\Omega \times(0, T), \Sigma=\Gamma \times(0, T)$. Define

$$
\begin{aligned}
F(y, u)= & \int_{\Omega} \gamma_{0}(x, y(x, T)) d x+\int_{\Omega} \psi_{0}(x, y(x, T)) d \mu_{0}(x) \\
& +\int_{Q} f_{0}(x, t, y(x, t), u(x, t)) d x d t \\
F_{j}(y, u)= & \int_{Q} \psi_{j}(x, t, y(x, t)) d \mu_{j}(x, t)+\int_{Q} f_{j}(x, t, y(x, t), u(x, t)) d x d t
\end{aligned}
$$

$j=1, \ldots, m$. We assume again that the functions $\psi_{j}, f_{j}$, and $\gamma_{j}$ are of class $C^{2}$ on $\bar{Q} \times \mathbb{R}$ and $\bar{Q} \times \mathbb{R}^{2}$, respectively. Moreover, real Borel measures $\mu_{j}, j=0, \ldots, m$, are given on $\Omega$ and $Q$, respectively. Now $\mu$ is the Lebesgue measure on $\mathrm{Q}, d \mu=d x d t$. 
Consider the optimal control problem

$(\mathrm{PAR})$

$$
\begin{cases}\operatorname{minimize} F(y, u) & \\ \frac{\partial y}{\partial t}-\Delta y+b(x, t, y, u)=0 & \text { in } Q \\ \partial_{\nu} y=0 & \text { on } \Sigma \\ y(x, 0)=0 & \text { in } \Omega \\ u_{a}(x, t) \leq u(x, t) \leq u_{b}(x, t) & \text { a.e. on } Q \\ F_{j}(y, u)=0, & 1 \leq j \leq m_{1} \\ F_{j}(y, u) \leq 0, & m_{1}+1 \leq j \leq m\end{cases}
$$

In this setting, the distributed control $u$ is looked upon in the space $L^{\infty}(Q)$; hence we set $X=Q$. The state $y$ belongs to $Y=\left\{y \in W(0, T) \mid y(0)=0, y_{t}-\Delta y \in\right.$ $\left.L^{q}(Q), \partial_{\nu} y \in L^{p}(\Sigma)\right\}$, where $q>N / 2+1$ and $p>N+1$ are given fixed. It is known that $Y \subset C(\bar{Q})$, the embedding being continuous for the graph norm. Assume that $b=b(x, t, y, u)$ satisfies the same conditions as the $f_{j}$. Additionally, we require that $\partial b / \partial y(x, t, y, u) \geq 0$ on $Q \times \mathbb{R} \times\left[\min u_{a}, \max u_{b}\right]$. Define

$$
A: Y \rightarrow L^{q}(Q) \times L^{p}(\Sigma) \quad \text { and } \quad B: Y \times L^{\infty}(Q) \rightarrow L^{q}(Q) \times L^{p}(\Sigma)
$$

by

$$
A y=\left(\begin{array}{c}
\frac{\partial y}{\partial t}-\Delta y \\
\partial_{\nu} y
\end{array}\right) \quad \text { and } \quad B(y, u)(x, t)=\left(\begin{array}{c}
b(x, t, y(x, t), u(x, t)) \\
0
\end{array}\right)
$$

The equation $A y+B(y, u)=0$, which is equivalent to our parabolic initial-boundary value problem, admits for each $u \in L^{\infty}(Q)$ exactly one solution $y \in Y$. We refer to [5]. The mapping $u \mapsto y$ is of class $C^{2}$ from $L^{\infty}(Q)$ to $Y$. Here, the Lagrange function is

$$
\begin{aligned}
\mathcal{L}(y, u, \varphi, \lambda)= & F(y, u)-\int_{Q}\left(y_{t}-\Delta y-b(x, t, y, u)\right) \varphi d x d t \\
& -\int_{\Sigma} \partial_{\nu} y \varphi d S d t+\sum_{j=1}^{m} \lambda_{j} F_{j}(y, u)
\end{aligned}
$$

where $\varphi$ is the adjoint state and $d S$ again denotes the Lebesgue surface measure induced on $\Gamma$. Equation (4.5) turns out to be the adjoint equation

$$
\left\{\begin{aligned}
-\frac{\partial \varphi}{\partial t}-\Delta \varphi+\frac{\partial b}{\partial y} \varphi & =\frac{\partial f_{0}}{\partial y}+\sum_{j=1}^{m} \bar{\lambda}_{j}\left(\frac{\partial f_{j}}{\partial y}+\frac{\partial \psi_{j}}{\partial y} \mu_{j}\right) & & \text { in } Q \\
\partial_{\nu} \varphi & =0 & & \text { in } \Sigma \\
\varphi(x, T) & =\frac{\partial \gamma_{0}}{\partial y}(x, \bar{y}(x, T))+\frac{\partial \psi_{0}}{\partial y}(x, \bar{y}(x, T)) \mu_{0} & & \text { in } \Omega
\end{aligned}\right.
$$

(all partial derivatives taken at $(x, \bar{y}, \bar{u})$ ). This equation has a unique solution $\bar{\varphi} \in$ $W^{1, s}(\Omega)$ associated with $(\bar{y}, \bar{u}, \bar{\varphi}, \bar{\lambda})$. If, however, $\psi_{j}=0, j=1, \ldots, m$, then $\bar{\varphi}$ is more regular, $\bar{\varphi} \in W(0, T) \cap C(\bar{Q})$. 
The relevant derivatives of $\mathcal{L}$ are

$$
\begin{aligned}
& \frac{\partial \mathcal{L}}{\partial u}(\bar{y}, \bar{u}, \bar{\varphi}, \bar{\lambda}) h \\
&= \int_{Q}\left[\frac{\partial f_{0}}{\partial u}(x, \bar{y}, \bar{u})+\sum_{j=1}^{m} \bar{\lambda}_{j} \frac{\partial f_{j}}{\partial u}(x, \bar{y}, \bar{u})-\bar{\varphi} \frac{\partial b}{\partial u}(x, \bar{y}, \bar{u})\right] h d x d t \\
&= \int_{Q} d(x, t) h(x, t) d x d t \\
& \mathcal{L}_{(y, u)}^{\prime \prime}(\bar{y}, \bar{u}, \bar{\varphi}, \bar{\lambda})\left[\left(y_{1}, h_{1}\right),\left(y_{2}, h_{2}\right)\right] \\
&=\int_{Q}\left(y_{1}, h_{1}\right)\left[f_{0}^{\prime \prime}(x, \bar{y}, \bar{u})+\sum_{j=1}^{m} \bar{\lambda}_{j} f_{j}^{\prime \prime}(x, \bar{y}, \bar{u})-\bar{\varphi} b^{\prime \prime}(x, \bar{y}, \bar{u})\right]\left(y_{2}, h_{2}\right)^{\top} d x d t \\
& \quad+\int_{\Omega} \frac{\partial^{2} \psi_{0}}{\partial y^{2}}(x, \bar{y}(T)) y_{1}(T) y_{2}(T) d \mu_{0}+\int_{Q} \sum_{j=1}^{m} \bar{\lambda}_{j} \frac{\partial^{2} \psi_{j}}{\partial y^{2}}(x, \bar{y}) y_{1} y_{2} d \mu_{j} \\
& \quad+\int_{\Omega} \frac{\partial^{2} \gamma_{0}}{\partial y^{2}}(x, \bar{y}(T)) y_{1}(T) y_{2}(T) d x .
\end{aligned}
$$

The first- and second-order conditions for the parabolic case are covered by Corollaries 4.1-4.3. We have to substitute $Q$ for $X$ there and replace the variable $x$ by $(x, t)$. Moreover, in the second-order conditions, $y$ and $h$ are coupled through the linearized initial-boundary value problem

$$
\left\{\begin{array}{l}
y_{t}-\Delta y+\frac{\partial b}{\partial y}(x, t, \bar{y}, \bar{u}) y+\frac{\partial b}{\partial u}(x, t, \bar{y}, \bar{u}) h=0 \\
\partial_{\nu} y=0 \\
y(x, 0)=0 .
\end{array}\right.
$$

We leave the calculations of the derivatives in (4.14) to the reader; they are obtained by an obvious modification of (4.28). We should mention again that these optimality conditions are meaningful only if the assumptions (A1)-(A3) are satisfied.

5. Verification of the assumptions. Our theory relies on the general assumptions (A1)-(A3). We shall see that (A1)-(A3) are naturally satisfied for the problem (ODE), while the situation is more complicated in the case of the elliptic or parabolic PDE.

(i) Problem (ODE). (A1). It is obviously sufficient to look at one of the functionals $G_{j}(u)=F_{j}(G(u), u)$ to assess the situation. We have

$$
G_{j}^{\prime}(\bar{u}) h=\int_{0}^{T} \frac{\partial f_{j}}{\partial y}(t, \bar{y}, \bar{u}) y d t+\int_{0}^{T} \frac{\partial f_{j}}{\partial u}(t, \bar{y}, \bar{u}) h d t,
$$

where $y=G^{\prime}(\bar{u}) h$. Here, $\partial f_{j} / \partial y, \partial f_{j} / \partial u$ are bounded and measurable functions. Moreover, the estimate

$$
\|y\|_{C[0, T]}=\left\|G^{\prime}(\bar{u}) h\right\|_{C[0, T]} \leq c\|h\|_{L^{2}(0, T)}
$$

holds, since $\|y\|_{C[0, T]} \leq c\|y\|_{H^{1}(0, T)} \leq c\|h\|_{L^{2}(0, T)}$. Thus the mapping $h \mapsto G_{j}^{\prime}(\bar{u}) h$ defines a linear and continuous functional on $L^{2}(0, T)$. By the Riesz representation 
theorem,

$$
G_{j}^{\prime}(\bar{u}) h=\int_{0}^{T} g_{j}(t) h(t) d t
$$

must hold with some $g_{j} \in L^{2}(0, T)$; hence (A1) is fulfilled.

(A2). Here, the derivative

$$
G_{j}^{\prime \prime}(\bar{u})\left[h_{1}, h_{2}\right]=\int_{0}^{T}\left(y_{1}, h_{1}\right) f_{j}^{\prime \prime}(t, \bar{y}, \bar{u})\left(y_{2}, h_{2}\right)^{\top} d t
$$

is characteristic for the discussion. All entries of $f_{j}^{\prime \prime}$ are bounded and measurable. If $h_{i}^{k} \rightarrow h_{i}$ in $L^{2}(0, T), k \rightarrow \infty, i=1,2$, then $y_{i}^{k} \rightarrow y_{i}$ in $C[0, T]$; hence $G_{j}^{\prime \prime}(\bar{u})\left[h_{1}^{k}, h_{2}^{k}\right] \rightarrow$ $G_{j}^{\prime \prime}(\bar{u})\left[h_{1}, h_{2}\right]$. This shows (A2).

(A3). First, we must estimate differences of the type $G_{j}^{\prime \prime}(\tilde{u})-G_{j}^{\prime \prime}(\bar{u})$ for $\tilde{u}$ in a $L^{\infty}$-neighborhood of $\bar{u}$. We get

$$
\left|\left(G_{j}^{\prime \prime}(\tilde{u})-G_{j}^{\prime \prime}(\bar{u})\right) h^{2}\right| \leq \int_{0}^{T}\left|f_{j}^{\prime \prime}(t, \tilde{y}, \tilde{u})-f_{j}^{\prime \prime}(t, \bar{y}, \bar{u})\right||(y, h)|^{2} d t
$$

where $\tilde{y}=G(\tilde{u}), \bar{y}=G(\bar{u}), y=G^{\prime}(\bar{u}) h$. Due to our assumptions, we find that

$$
\left|\left[G_{j}^{\prime \prime}(\tilde{u})-G_{j}^{\prime \prime}(\bar{u})\right] h^{2}\right| \leq \delta\left(\|y\|_{C[0, T]}^{2}+\|h\|_{L^{2}(0, T)}^{2}\right) \leq c \delta\|h\|_{L^{2}(0, T)}^{2},
$$

where $\delta \rightarrow 0$ as $\|\tilde{u}-\bar{u}\|_{L^{\infty}} \rightarrow 0$. Another characteristic part in $\partial^{2} L / \partial u^{2}$ is the coupling of the nonlinearity $b$ with $\bar{\varphi}$. It is the essential advantage of our simplified case (ODE) that $\bar{\varphi} \in L^{\infty}(0, T)$. Therefore, we are justified to estimate

$$
\begin{aligned}
\left|\int_{0}^{T}(y, h) b^{\prime \prime}(t, \bar{y}, \bar{u})(y, h)^{\top} \bar{\varphi} d t\right| & \leq c\|\bar{\varphi}\|_{L^{\infty}(0, T)}\left(\|y\|_{C[0, T]}^{2}+\|h\|_{L^{2}(0, T)}^{2}\right) \\
& \leq c\|h\|_{L^{2}(0, T)}^{2} .
\end{aligned}
$$

Discussing all second-order terms in this way, we easily verify that (A3) is also satisfied.

(ii) Elliptic problem (ELL). We repeat the discussion of (A1)-(A3) along the lines of (i) but concentrating on the essential differences with the case of (ODE). Here, it holds that

$$
\begin{aligned}
G_{j}^{\prime}(\bar{u}) h= & \int_{\Omega} \frac{\partial \gamma_{j}}{\partial y}(x, \bar{y}) y d x+\int_{\Omega} \frac{\partial \psi_{j}}{\partial y}(x, \bar{y}) y d \mu_{j} \\
& +\int_{\Gamma} \frac{\partial f_{j}}{\partial y}(x, \bar{y}, \bar{u}) y d S+\int_{\Gamma} \frac{\partial f_{j}}{\partial u}(x, \bar{y}, \bar{u}) h d S,
\end{aligned}
$$

where $y=G^{\prime}(\bar{u}) h$. In contrast to (5.2), now the mapping $G^{\prime}(\bar{u})$ is not in general continuous from $L^{2}(\Gamma)$ to $C(\bar{\Omega})$. This property only holds for $N=\operatorname{dim} \Omega=2$ (see [9]). For $N>2$ we assume that $\Omega_{j}$, the support of $\mu_{j}$, satisfies $\bar{\Omega}_{j} \subset \Omega$. Then the mapping $h \mapsto G^{\prime}(\bar{u}) h$ is continuous from $L^{2}(\Gamma)$ to $C\left(\bar{\Omega}_{j}\right)$; hence $h \mapsto G_{j}^{\prime}(\bar{u}) h$ is a linear and continuous functional on $L^{2}(\Gamma)$. The Riesz theorem yields a representation analogous to (5.3). Hence (A1) is shown under additional assumptions on the subdomains $\Omega_{j}$. (A2) then holds true in the same way. Notice that the restriction to $\Omega_{j}$ is not needed if all $\psi_{j}$ vanish. 
To verify (A3) we need even more restrictions on the data. The situation is easy if $\psi_{j}=0, j=1, \ldots, m$. Then all given data in the adjoint equation are bounded and measurable, and the regularity theory of elliptic equations yields $\bar{\varphi} \in C(\bar{\Omega})$. In this case, (A3) is obviously satisfied.

Let us now assume that at least one of the $\psi_{j}$ is not zero. Then the best regularity of the trace $\bar{\varphi}_{\mid \Gamma}$ is $\bar{\varphi}_{\mid \Gamma} \in L^{r}(\Gamma)$ for all $r<(N-1) /(N-2)$. For instance, $\varphi \in L^{r}(\Gamma)$ for all $r<\infty$ is obtained in the case $N=2$. We therefore cannot assume that $\bar{\varphi} \in L^{\infty}(\Omega)$. Regard the elliptic counterpart to (5.5),

$$
\begin{aligned}
\left|\int_{\Gamma}(y, h) b^{\prime \prime}(x, \bar{y}, \bar{u})(y, h)^{\top} \bar{\varphi} d S\right| & =\left|\int_{\Gamma} \bar{\varphi}\left(\frac{\partial^{2} b}{\partial y^{2}} y^{2}+2 \frac{\partial^{2} b}{\partial y \partial u} y h+\frac{\partial^{2} b}{\partial u^{2}} h^{2}\right) d S\right| \\
& \leq c \int_{\Gamma}\left(|\bar{\varphi}| y^{2}+|\bar{\varphi}| y h+|\bar{\varphi}| h^{2}\right) d S .
\end{aligned}
$$

This expression has to be estimated for $h \in L^{2}(\Gamma)$. If $\bar{\varphi}_{\mid \Gamma} \notin L^{\infty}(\Gamma)$, which is the normal case, then we must exclude the third term from (5.6). This means that $\partial^{2} b / \partial u^{2}$ has to disappear - $u$ must appear linearly. Next we consider the second term, where $\left\|\bar{\varphi}_{\mid \Gamma} y\right\|_{L^{2}(\Gamma)}$ is estimated against $\|h\|_{L^{2}(\Gamma)}$. The mapping $h \mapsto y$ is continuous from $L^{2}(\Gamma)$ to $C(\Gamma)(N=2)$, to $L^{r}(\Gamma)$ for all $r<\infty(N=3)$, and to $L^{r}(\Gamma)$ for all $r<2(N-1) /(N-3)(N>3)$. Therefore, the second term can be estimated iff $N=2$, while it must be cancelled for $N>2$. The latter means $\partial^{2} b / \partial u \partial y=0$-here $b=b_{1}(x, y)+b_{2}(x) u$ must hold. In the same way we arrive at the surprising fact that for $N>3$ the first term in (5.6) must vanish, too. In other words, in the case of elliptic boundary control with pointwise functionals $F_{j}$, we cannot admit nonlinear equations for $N>3$.

Remark 5.1. We should underline again that these restrictions are not needed if the functionals $F_{j}$ are sufficiently regular $\left(\psi_{j}=0, j=1, \ldots, m\right)$. Moreover, the case of distributed controls permits us to slightly relax the restrictions on the dimension $N$.

(iii) Parabolic problem (PAR). Once again, (A1)-(A3) are satisfied if $\psi_{j}=0$, $j=1, \ldots, m$. This is due to the high regularity $\bar{\varphi} \in W(0, T) \cap C(\bar{Q})$ in this case.

In the opposite case, the problem of regularity is even more delicate than in the elliptic problem. We cannot discuss the general case in detail and refer to the recent paper [22]. Instead of this, let us explain the point for a very particular constraint: Suppose that only one (pointwise) state constraint of the form

$$
g_{1}(y, u)=\int_{0}^{T} y\left(x_{1}, t\right) d t=0
$$

is given, where $x_{1} \in \Omega$ is a fixed position of observation. To make the theory work, we need some strong restrictions: We assume $N=\operatorname{dim} \Omega=1$, i.e., $\Omega=(a, b)$, and require that $\partial^{2} b / \partial u^{2}=0$ (the control appears linearly). Then the mapping $h \mapsto y=G^{\prime}(\bar{u}) h$ is continuous from $L^{2}(Q)$ to $C(\bar{Q})$, and the functional $h \mapsto g_{1}(y, h)$ is continuous on $L^{2}(Q)$. We know that $\bar{\varphi} \in L^{s}(Q)$ for all $s<3$. (This follows from Theorem 4.3 in [22] for $N=1$ and $\alpha=\tilde{\alpha}$.) Hence $\bar{\varphi} \notin L^{\infty}(Q)$, and that is the reason why we cannot admit a control appearing nonlinearly. The estimate of the parabolic counterpart of (5.6) is

$$
\begin{aligned}
& \left|\int_{Q}\left(\frac{\partial^{2} b}{\partial y^{2}} \bar{\varphi} y^{2}+2 \frac{\partial^{2} b}{\partial y \partial u} \bar{\varphi} y h\right) d x d t\right| \\
& \quad \leq c\|\bar{\varphi}\|_{L^{1}(Q)}\|y\|_{L^{\infty}(Q)}^{2}+c\|\bar{\varphi}\|_{L^{2}(Q)}\|y\|_{L^{\infty}(Q)}\|h\|_{L^{2}(Q)} \leq c\|h\|_{L^{2}(Q)}^{2} .
\end{aligned}
$$


Discussions of this type reveal that (A1)-(A3) are satisfied. However, we needed very strong assumptions, in particular $N=1$. The case $N=2$ can be handled under additional restrictions concerning the appearance of control and observations ("control and observations have disjoint supports"; see [22]).

If there are no pointwise state constraints, the situation is easier, as the reader can check.

Remark 5.2. The second-order conditions established in the previous sections allow us to study $L^{\infty}$-local solutions. This causes specific difficulties if the optimal control exhibits jumps. Therefore, $L^{p}$-optimality conditions can be more interesting. An associated extension to $L^{p}$ is possible, provided that the control-state mapping $u \mapsto y$ and the objective functional are differentiable from $L^{p}$ to $L^{\infty}$. Under associated restrictions (for instance, that the control appear linearly in the state equation and the cost functional be quadratic with respect to the control), this extension to $L^{p}$ is possible for sufficiently large $p<\infty$. For some associated results we refer the reader to Casas, Tröltzsch, and Unger [8] and Dunn [14].

Remark 5.3. For some optimal control problems, the second-order condition

$$
\frac{\partial^{2} L}{\partial u^{2}}(\bar{u}, \bar{\lambda}) h^{2}>0 \quad \forall h \in C_{\bar{u}}^{0} \backslash\{0\},
$$

along with a certain positivity of the second derivative with respect to the control of the Hamiltonian, provide sufficient optimality conditions. The reader is referred to Casas and Mateos [6], where these conditions are proved to be sufficient and equivalent to (3.26); see also Bonnans and Zidani [4]. In particular, if the control appears linearly in the state equation and the cost functional is quadratic and positive with respect to the control, then the above condition is sufficient for optimality.

\section{REFERENCES}

[1] N. Arada, E. Casas, and F. Tröltzsch, Error estimates for a semilinear elliptic control problem, Comput. Optim. Appl., to appear.

[2] W. Alt and K. Malanowski, The Lagrange-Newton method for nonlinear optimal control problems, Comput. Optim. Appl., 2 (1993), pp. 77-100.

[3] J. Bonnans AND E. CASAS, Contrôle de systèmes elliptiques semilinéaires comportant des contraintes sur l'état, in Nonlinear Partial Differential Equations and Their Applications. Collège de France Seminar, Vol. 8, H. Brezis and J. Lions, eds., Longman Scientific and Technical, New York, 1988, pp. 69-86.

[4] J.F. Bonnans AND H. ZIDANI, Optimal control problems with partially polyhedric constraints, SIAM J. Control Optim., 37 (1999), pp. 1726-1741.

[5] E. CASAs, Pontryagin's principle for state-constrained boundary control problems of semilinear parabolic equations, J. Anal. Appl., 15 (1996), pp. 687-707.

[6] E. Casas And M. Mateos, Second order optimality conditions for semilinear elliptic control problems with finitely many state constraints, SIAM J. Control Optim., 40 (2002), pp. 1431-1454.

[7] E. Casas and F. Tröltzsch, Second order necessary optimality conditions for some stateconstrained control problems of semilinear elliptic equations, Appl. Math. Optim., 39 (1999), pp. 211-227.

[8] E. Casas, F. Tröltzsch, and A. Unger, Second order sufficient optimality conditions for a nonlinear elliptic control problem, J. Anal. Appl., 15 (1996), pp. 687-707.

[9] E. Casas, F. Tröltzsch, And A. Unger, Second order sufficient optimality conditions for some state-constrained control problems of semilinear elliptic equations, SIAM J. Control Optim., 38 (2000), pp. 1369-1391.

[10] F. Clarke, A new approach to Lagrange multipliers, Math. Oper. Res., 1 (1976), pp. 165-174.

[11] A.L. Dontchev, W.W. Hager, A.B. Poore, And B. Yang, Optimality, stability, and convergence in nonlinear control, Appl. Math. Optim., 31 (1995), pp. 297-326. 
[12] J.C. Dunn, Second-order optimality conditions in sets of $L^{\infty}$ functions with range in a polyhedron, SIAM J. Control Optim., 33 (1995), pp. 1603-1635.

[13] J. DunN, On second-order sufficient optimality conditions for structured nonlinear programs in infinite-dimensional function spaces, in Mathematical Programming with Data Perturbations, A. Fiacco, ed., Marcel Dekker, New York, 1998, pp. 83-107.

[14] J.C. DunN, $\mathbb{L}^{2}$ sufficient conditions for end-constrained optimal control problems with inputs in a polyhedron, SIAM J. Control Optim., 36 (1998), pp. 1833-1851.

[15] W.W. HAGER, Error bounds for Euler approximation of a state and control constrained optimal control problem, Numer. Funct. Anal. Optim. 21 (2000), pp. 653-682.

[16] M.R. Hestenes, Calculus of Variations and Optimal Control Theory, John Wiley, New York, 1966.

[17] A.D. Ioffe, Necessary and sufficient conditions for a local minimum. 3: Second order conditions and augmented duality, SIAM J. Control Optim., 17 (1979), pp. 266-288.

[18] K. Ito AND K. Kunisch, Augmented Lagrangian-SQP methods for nonlinear optimal control problems of tracking type, SIAM J. Control Optim., 34 (1996), pp. 874-891.

[19] H. MAURER, First and second-order sufficient optimality conditions in mathematical programming and optimal control, Math. Programming Study, 14 (1981), pp. 163-177.

[20] H. MaURer AND J. Zowe, First- and second-order conditions in infinite-dimensional programming problems, Math. Programming, 16 (1979), pp. 98-110.

[21] H.D. Mittelmann, Verification of second-order sufficient optimality conditions for semilinear elliptic and parabolic control problems, Comput. Optim. Appl., 20 (2001), pp. 93-110.

[22] J.-P. Raymond And F. Tröltzsch, Second order sufficient optimality conditions for nonlinear parabolic control problems with state-constraints, Discrete Contin. Dynam. Systems, 6 (2000), pp. 431-450.

[23] V. Schulz, ED., SQP Based Direct Discretization Methods for Practical Optimal Control Problems, J. Comput. Appl. Math., special issue, 120 (2000). 